\title{
NASA Human Spaceflight Architecture Team Lunar Destination Activities
}

\author{
J. F. Connolly ${ }^{1}$, R. P. Mueller ${ }^{2}$ and R. J.Whitley ${ }^{3}$
}

${ }^{1}$ Exploration Missions \& Systems Office, National Aeronautics \& Space Administration (NASA) Johnson Space Center, 2101 NASA Parkway Houston, Texas 77058, USA; PH (281) 483-4881; email: john.connolly@nasa.gov

${ }^{2}$ Surface Systems Office, National Aeronautics \& Space Administration (NASA). Mail Stop: NE-S, Kennedy Space Center, KSC, FL 32899, USA; PH (321)-867-2557; email: rob.mueller@nasa.gov

${ }^{3}$ Exploration Missions \& Systems Office, National Aeronautics \& Space Administration (NASA) Johnson Space Center, 2101 NASA Parkway Houston, Texas 77058, USA; PH (281) 483-483-9577; email: ryan.j.whitley@nasa.gov

\section{ABSTRACT}

NASA's Human Spaceflight Architecture Team (HAT) Lunar Destination Team has been developing a number of "Design Reference Missions" (DRM) to inform exploration architecture development, transportation approaches, and destination elements and operations. There are four destinations being considered in the HAT studies: Cis-Lunar, Lunar, Near Earth Asteroids and Mars. The lunar destination includes all activities that occur on the moon itself, but not low lunar orbit operations or Earth Moon LaGrange points which are the responsibility of the HAT Cis-Lunar Team.

This paper will review the various surface DRMs developed as representative scenarios that could occur in a human lunar return. The approaches have been divided into two broad categories: a seven day short stay mission with global capabilities and a longer extended duration stay of 28 days which is limited to the lunar poles as a landing zone. The surface elements, trade studies, traverses, concept of operations and other relevant issues and methodologies will be presented and discussed in the context and framework of the HAT ground rules and assumptions which are constrained by NASA's available transportation systems. An international collaborative effort based on the 2011 Global Exploration Roadmap (GER) will also be examined and evaluated.

\section{INTRODUCTION}

Since 1969, NASA has conducted numerous internal studies and chartered 24 blue ribbon panels to assess future human space flight strategy, and exploration concepts and technologies. Following the cancellation of NASA's Constellation program in 2010, the most current instantiations of these groups examined a broad trade space of program strategies and technical approaches in an effort to meet priorities from the White House, Congress, and other stakeholders. In 
light of findings that its Constellation program was "pursuing goals that do not match allocated resources" (Augustine et al), new affordability options were examined. A major conclusion was that the NASA Human Space Flight (HSF) architecture must provide the flexibility to accommodate technical, programmatic, economic and political dynamics while enabling a safe, affordable and sustainable human space exploration program.

In an attempt to satisfy the uncertainties and conflicting stake holder positions, NASA's Human Exploration Framework Team (HEFT) selected a "Capability Driven Framework" (CDF) that emphasized the development of capabilities that would enable stepwise missions to ever more-difficult destinations, rather than the previous NASA strategy where a destination was given and the capabilities defined to attain this destination. The CDF builds capabilities that could incrementally enable many potential paths to GEO, L1/L2, the lunar surface, Near-Earth Asteroids (NEAs), Martian moons, and the surface of Mars.

In early 2011, the Human Spaceflight Architecture Team (HAT) took the reins from the HEFT to provide strategic guidance to human space exploration planning. The HAT serves as the NASA agency decision support focal point with primary responsibility for integrated and cross-cutting strategic analysis of human space exploration for NASA's decision makers. HAT's scope includes integrated development and assessment of architectures, systems, mission scenarios, and concepts of operation across the human and robotic space exploration spectrum. The team began to refine Design Reference Missions (DRMs) to generate an integrated capability-driven approach for systems planning within a multi-destination framework. HAT has also identified and assessed technology and capabilities needs and priorities consistent with the evolution of the architecture and perform robust cost analyses to assess overall affordability, viability, and sustainability

\section{DESTINATION FOCUS}

HAT identified the need to balance transportation studies with a sense of what could be accomplished at various destinations in space. As a result, HAT Destination Teams were chartered to bring a destination context to the Capability Driven Framework and develop a destination companion to the transportation DRM analyses. The Cis-lunar, lunar surface, Near-Earth Asteroid and Mars destination teams were instructed to focus on what to do at the destinations enabled by HAT's Design Reference Missions (DRMs) and to work closely with DRM teams to balance destination operations and transportation constraints. The destination teams were also to organize and coordinate the development of data about human's activities occurring at each destination, to maintain cognizance of all past studies and missions, and all current events relative to each destination. HAT's destination leads also became the team's primary interface with destination-affiliated Agency and external groups such as the Lunar Exploration 
Analysis Group (LEAG), Mars Exploration Program Analysis Group (MEPAG) and Small Bodies Assessment Group (SBAG).

HAT's destination teams each examined the critical decisions that define the character of destination operations, and often illustrated these decisions in the form of a decision/trade "tree". Down selecting candidate destination missions from this tree, the destination teams expanded the transportation DRM mission descriptions ("bat charts") to now include surface operations and elements. Each team created a series of "Street View" charts that detailed the destination operations for each transportation DRM and matched them with detailed Ops Con/Ops Timelines. In the process of defining the surface mission in greater detail, the teams also gave further definition to destination payloads, defined required new Elements and identified impacts to transportation DRMs.

\section{LUNAR DESTINATION TRADE TREE}

In order to do a comprehensive assessment of all DRM options that are possible, the Lunar Destination Team pursued a six dimensional matrix which covers all options whether they are desirable or feasible or not. Then through a process of logical analysis the trade tree was trimmed down by eliminating branches with obvious inconsistencies. What remains is a drastically reduced trade space which can then be used as the basis for comparison to missions already flown or for future DRM studies and further detailed analysis by "drilling down".

\section{Building the Trade Tree}

The lunar trade tree was developed from a brainstorming exercise based on what could drive the need for a given functional capability for a crewed mission to the moon. The focus was on driving out all possibilities, regardless of heritage. After the trade tree was formulated, previously flown mission architectures (Apollo) and previously designed project plans (Constellation) were then aligned with the appropriate branches.

The trade tree was built by assembling relevant categories for a general capability human mission to the moon. The full lunar trade tree is shown in Figure 1. While the alternatives are dependent on technological capability, the categories themselves come from a function-driven framework. Regardless of the specific exploration goals for a given mission, the basic lunar human mission need is to sustain the crew for the duration of the mission and provide an acceptable amount of mobility for the crew to perform a set of exploration activities, whatever they may be. The first two categories can then be defined from each half of this overall need as "Surface Duration" and "Mobility Range." 


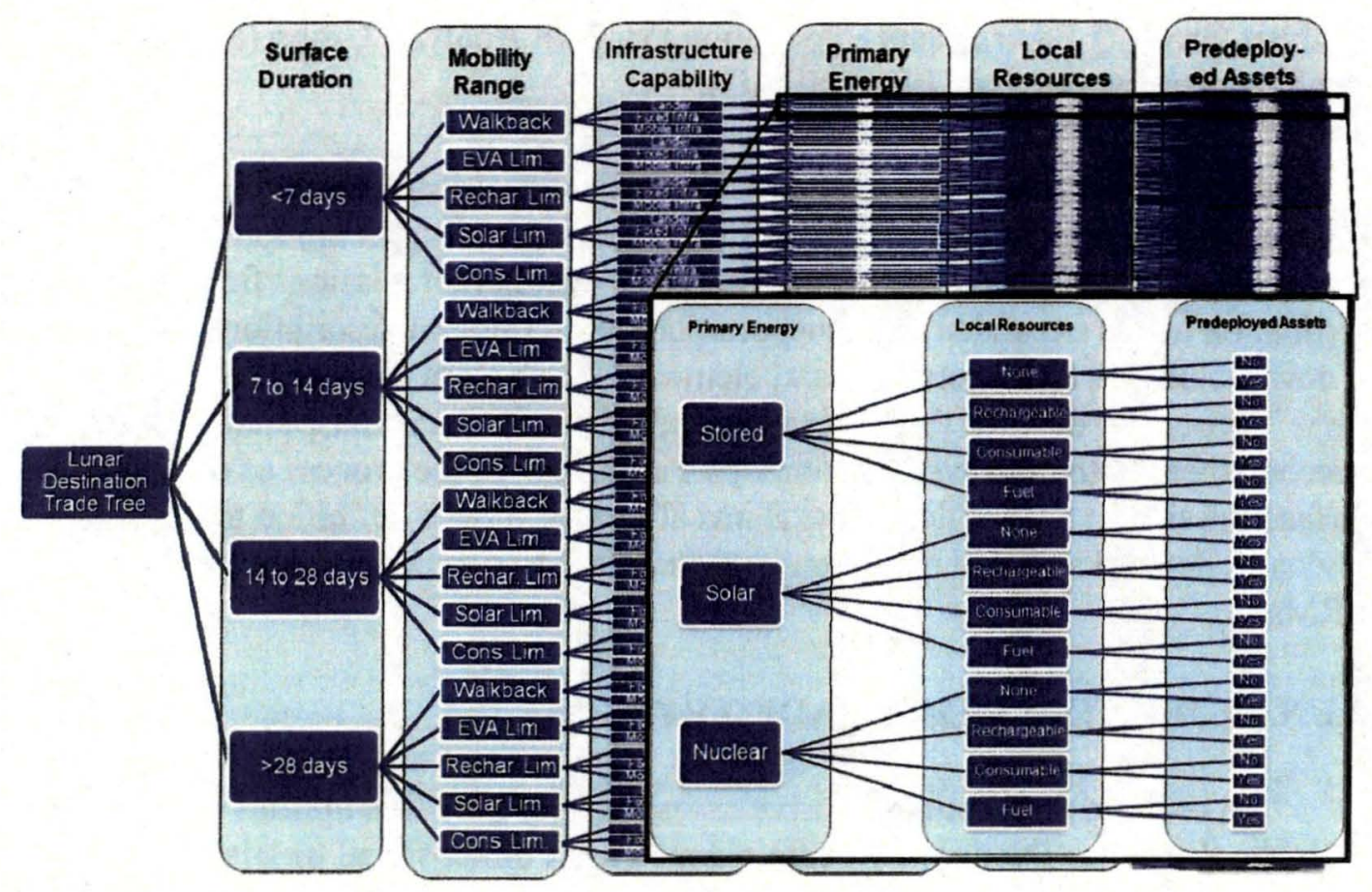

Figure 1: Full Lunar Trade Tree

In order for a system to provide surface stay capability it will, by definition, have certain quantities of mass, volume and power. While mass, volume, and power may be the most basic building blocks, functions that tie these mission driving parameters to physical hardware are more helpful as categories. As such, mass and volume needs are translated into habitability and reusability needs, which in turn can are given as two more categories for the lunar trade tree: "Infrastructure Capability" and "Use of Local Resources" respectively. Power is tied directly to the fifth category, "Primary Energy." All of these categories have mass, volume, and power implications that address various levels of surface stay capability. The final category is primarily related to mission planning rather than surface mission needs. Some mission architectures logistically require large mass and volume payloads that require predeployment. Thus, the sixth and final category is "Pre-deployed Assets."

Once the categories were determined, the fields were rather straight-forward to populate, as shown in Figure 2, which each category having a different number of fields. To get the total number of possible branches, the number of fields were multiplied together, which resulted in a grand total of 1440 possible cases. 
Surface Duration:

$<7$ Days - Akin to a sortie mission

$7-14$ days - Time represented by lunar daylight

14 - 28 days - A lunar synodic day

$>28$ days - Longer than a lunar synodic day

Infrastructure Capability:

Lander Only - Fixed site

Fixed Infrastructure - Surface habitat remains in place

Mobile Infrastructure - Surface habitat is mobile

Use of Local Resources:

None - Stand alone

Re-chargeable Elements - Solar energy as ISRU

ISRU for Consumables - Small prod. quan.

ISRU for Propellants - Large production quan.
Mobility Range:

Walkback - Max $10 \mathrm{~km}$ EVA walk back, eg. 1 rover

EVA Limited - Max $15 \mathrm{~km}$ contin. return, eg. 2 rovers

Range Limited by Power - E.g. fixed recharge station

Range Limited by Solar Power $\mathbf{7}$ day out 7 day back

Range Limited by Consumables - Energy Unlimited

\section{Primary Energy Generator:}

Stored - Stored Energy, E.g. batteries, fuel cells

Solar - Solar Energy, E.g. solar cells

Nuclear - Nuclear Power, local source of power

\section{Predeployed Assets:}

No - No predeployed assets

Yes - Predeployed assets exist

Figure 2: Field Breakdown of Lunar Trade Tree Categories

\section{Ruling Out Logical Disconnects in Trade Tree}

While the categorization process created 1440 possible branches, not every branch can or should be a real mission. Thus, a trade trimming process was implemented. In order to remain unbiased, only those cases that are truly illogical were removed. The so-called logical disconnects stem from inconsistencies between specific fields from one category to the next. To simplify the elimination process, a 2-D trade tree matrix was constructed, where every field from a given category was weighed against every field from every other category. This matrix is shown in Figure 3.

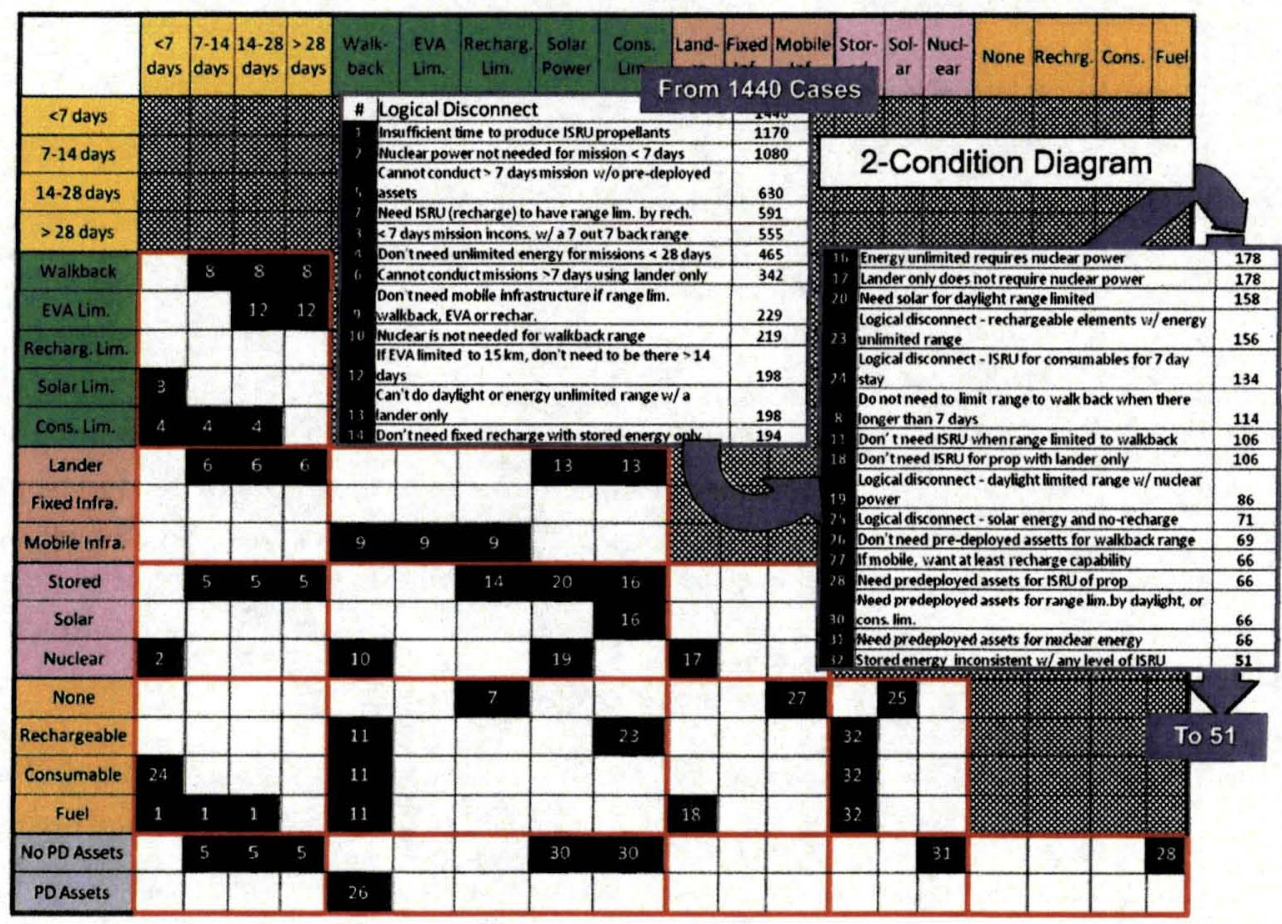

Figure 3: Elimination of Branches from Lunar Trade Tree 
It is not surprising that inconsistencies exist. While the categories were based on specific functional capability, the fields are largely interdependent, especially since the fields were designed with increasing capability to flush out all possible combinations.

An individual 2-D logical disconnect on its own has the ability to eliminate a large swath of branches, as illustrated in

Figure 3. Each category versus category matrix element is represented by a large red box of squares including all possible combinations of fields between those two categories. The grayed out region represents redundant branches of the trade space, as order does not matter. The diagonal of the matrix is also grayed out since a category cannot be evaluated against itself. If every possibility within a single red box is eliminated, then all 1440 branches are eliminated. For example, in the upper left hand corner of

Figure 3 there is red box representing the surface duration vs. mobility range fields. Logical disconnects numbered 3, 4, 8 and 12 eliminate specific cross-field cases from the tree. Assuming no other branches from any other categories were eliminated (all the other boxes were blank throughout the matrix), 9 blacked out boxes out of a possible 20 boxes means that 648 cases are removed from the lunar trade tree. When other logical disconnects from other red boxes are added, the interdependencies must be accounted for. Thus, as more logical disconnects are found, fewer cases are removed. In the end, for all 28 logical disconnects listed, the total number of viable cases reduces from 1440 to 51 .

\section{Understanding the Remaining Cases}

With more than $96 \%$ of the trade tree eliminated, the final 51 cases can be scrutinized in more detail. The trade tree itself results in branches that have heritage in Apollo or Constellation or other studies. The cases already flown (Apollo) are on the shortest duration and limited capability extreme while many of the future mission designs are at the long duration and high capability extreme. This leaves room for new cases in-between these extremes for future study.

In order to better understand the remaining cases, each branch was assigned physical components and laid out individually in mini "street view" diagrams. Street view is a term used by the HAT to designate activities at the destination itself in a detailed graphical synopsis. These individual diagrams were then placed into groups to identify cases worth further study.

Figure 4 provides a legend for the building blocks used in each category and some example street view diagrams for heritage missions. Many of the categories have crossphysical hardware implications, so the individual components were designed to be modular. Also, the diagrams are at the "stick-figure" level and should be treated as models to be populated with additional design analysis, as needed. 
Figure 5 articulates a few new branches that are available for further study. Most of the remaining branches are in the $>28$ day category since this surface duration offers the most flexibility in capability. However, there are many intermediate 7-14 day and 1428 day missions worth diving deeper into.

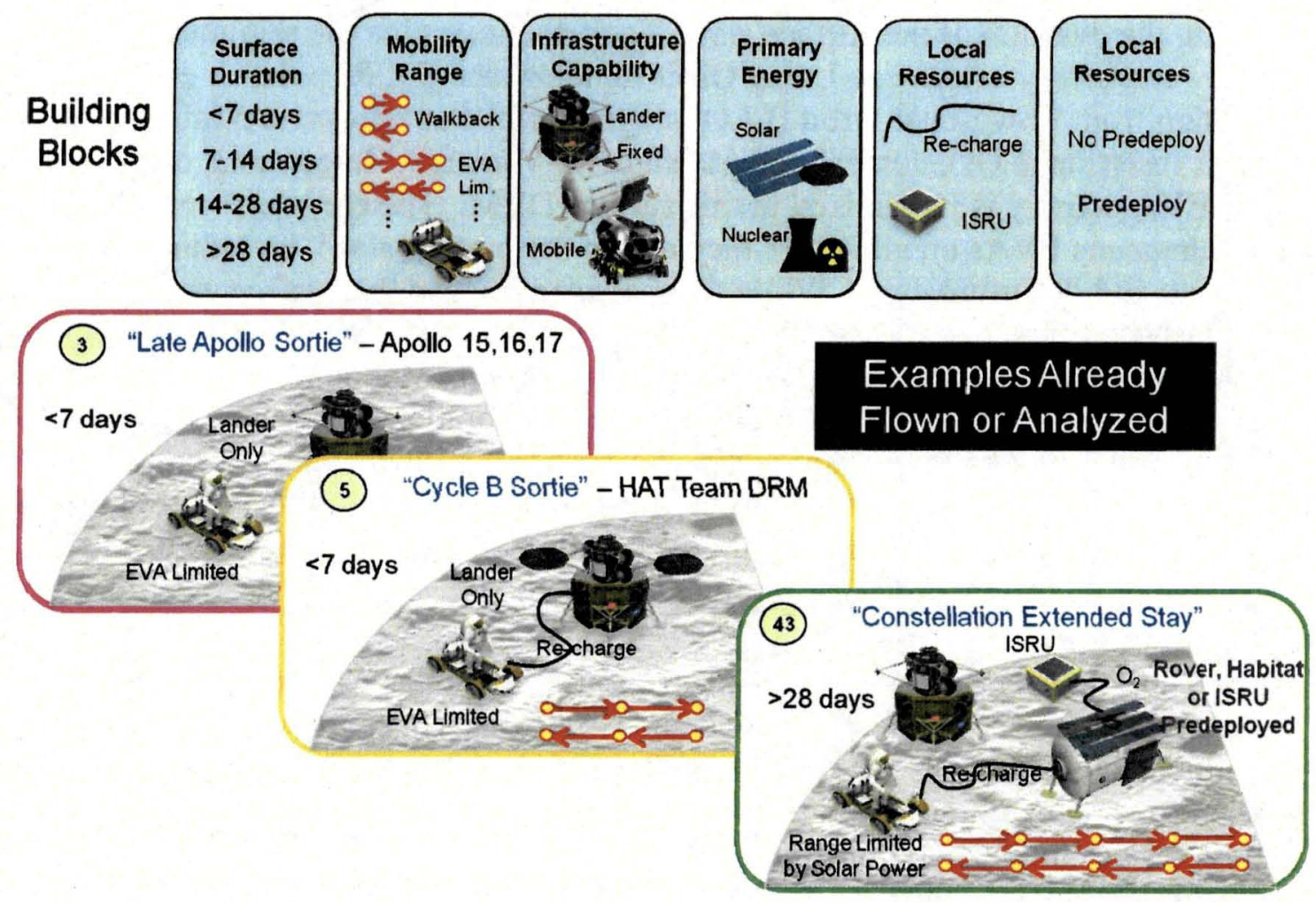

Figure 4: Example Street View Diagrams of Heritage Missions

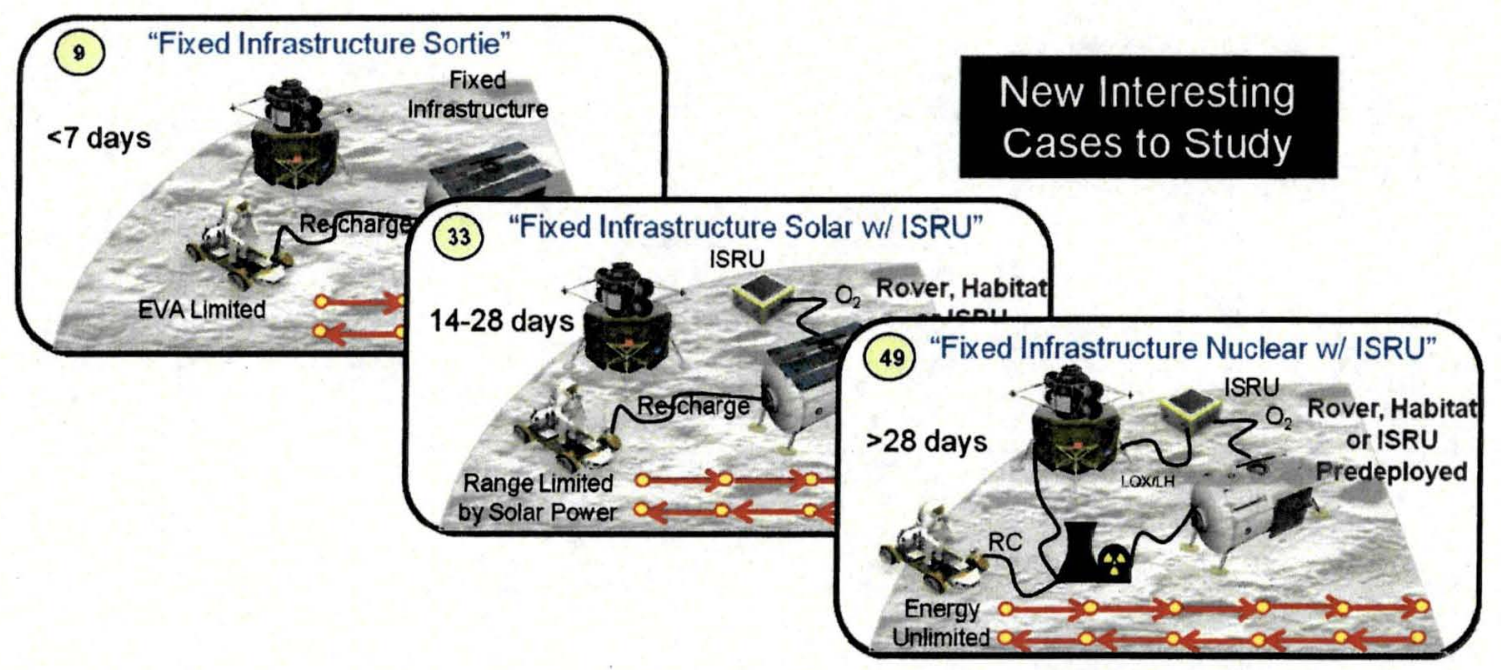

Figure 5: Example Street View Diagrams of new Lunar Surface Mission Possibilities 


\section{SORTIE HUMAN LUNAR MISSION}

On one extreme of the continuum of lunar surface missions is the lunar sortie mission. Sortie missions, by definition, do not utilize any significant pre-emplaced elements or logistics, and are conducted with the crew living out of the lander during the duration of the surface stay. To further examine the minimum hardware/energy extreme, a Lunar Orbit Rendezvous (LOR) polar or equatorial mission from Low Lunar Orbit (LLO) staging orbit was chosen to minimize Lunar Orbit Insertion (LOI), descent and ascent delta- $\mathrm{V}$ energy. In contrast, crew size and EVA activity is maximized in this surface DRM. The crew of 4 conducts simultaneous EVAs on all 7 days they are on the lunar surface, including EVAs on landing and departure days. While the crew descends to the surface, the un-crewed MPCV is left in a low lunar orbit that "node walks" over the landing site, enabling a minimum energy planar descent and ascent. The crew conducts the descent and ascent in LES-type suits, and perform surface exploration in separate surface EVA suits that are pre-docked to suit locks. To further minimize mission complexity, the 7-day mission is conducted entirely during lunar "day", and is powered by solar arrays on the surface. The lander delivers $500 \mathrm{~kg}$ of cargo to the surface, and is capable of returning $250 \mathrm{~kg}$ of samples on ascent.

Short duration sortie missions emphasize Extra Vehicular Activity (EVA) for exploration science and technology demonstrations. This particular example simultaneously deploys all $4 \mathrm{crew}$ members on the lunar surface to maximize the scientific and exploration return for the mission. When not conducting EVAs, the crew resides in the lander habitation module, which provides all the routine functions of eating, sleeping, housekeeping, exercise, and personal hygiene. Intra Vehicular Activity (IVA) functions also include preparing and maintaining pressure suits, planning for subsequent operations on the lunar surface, and all activities associated with post-landing and pre-ascent operations.

\section{Transportation Architecture}

The sortie mission transportation DRM is shown in Figure 6. Two Space Launch System (SLS) launches are required to place the Orion Multi Purpose Crew Vehicle (MPCV) and lunar lander, respectively, into low lunar orbit in an inclination that allows for a minimum energy descent and ascent at the "bookends" of the 7-day surface mission. Each SLS launch uses a cryogenic upper stage to perform the trans-lunar injection maneuver, and then again to perform the lunar orbit insertion burn to place the lander, and then Orion, into lunar orbit. Following a 5-day coast to the moon, the crew arrives in lunar orbit and performs rendezvous and docking with the lander. After checking out and transferring to the lander, the crew descends to the surface to perform the surface mission. While on the surface, the crew lives out of the lander's habitation module which, with the descent stage, is left on the surface at the end of the mission. Following 7 days on the lunar 
surface, the crew returns to lunar orbit in the lander's ascent module and re-joins the Orion vehicle. After the transfer of crew and samples to Orion, the lander ascent stage is expended, and the service module performs the trans-Earth injection burn. Following a 5-day fall back to Earth, the Orion capsule performs a direct Earth entry and landing in the Pacific ocean to the west of California.

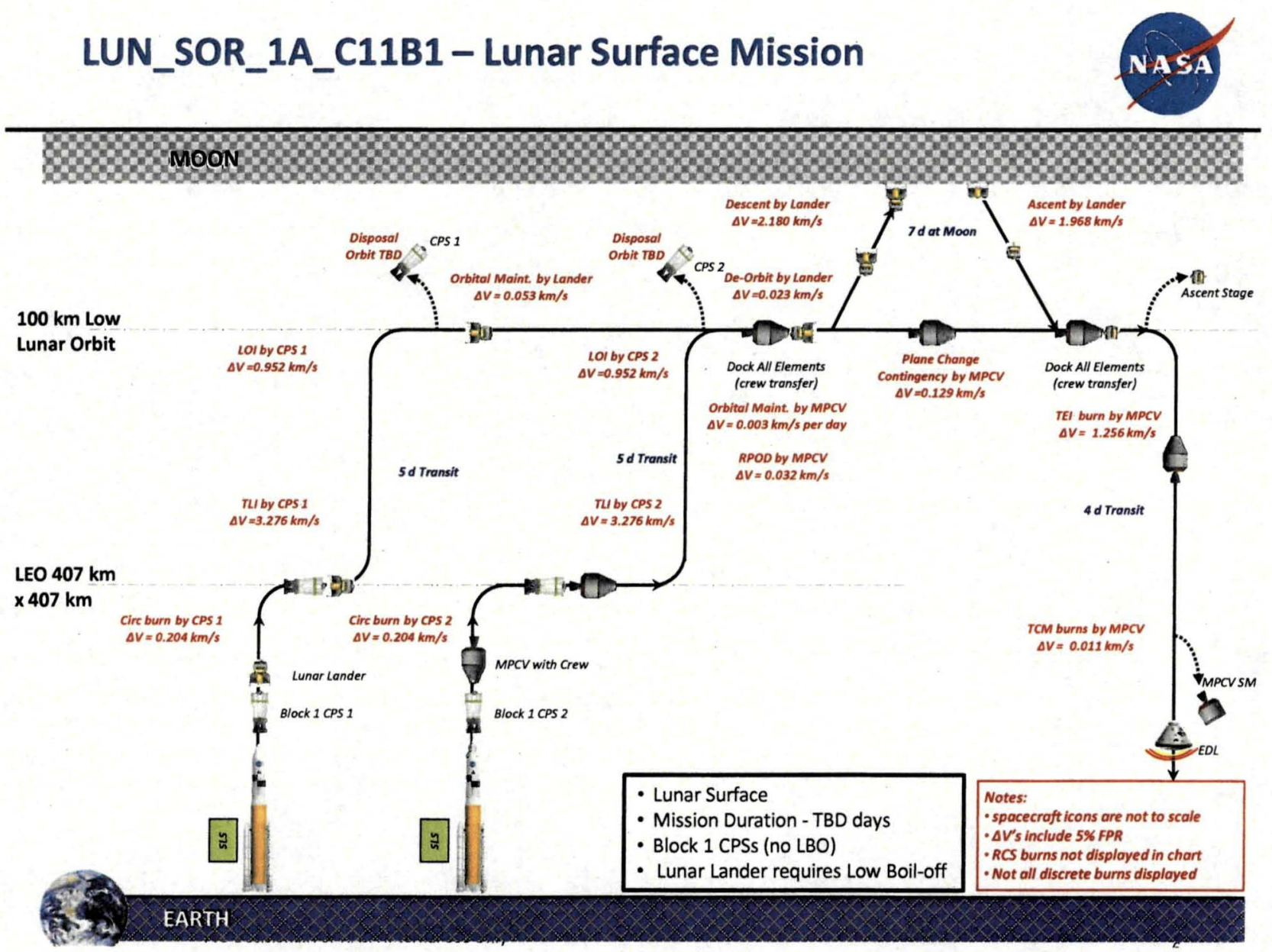

Figure 6. Lunar Sortie Mission Transportation DRM

\section{Surface Hardware}

This lunar sortie mission emphasizes geology and substantial rover traverses in order to investigate multiple geological units near the central peak of Tsiolkovsky Crater . This sortie mission also emphasizes a minimalist approach to science equipment, emphasizing diverse, substantial geologic sample return. To accomplish this sortie mission, two unpressurized rovers are deployed to give each pair of crewmembers the capability to rove up to $32 \mathrm{~km}$ roundtrip. Rovers are 
recharged between EVAs from the lander. Each rover carries a ground penetrating radar to map the subsurface structure and determine mare thickness during traverses. Rovers also carry a total of 4 instrument stations that will be deployed during selected traverses, and will continue to operate following the crew's departure. The instrument stations contain geophones, seismic sources, and surface magnetometers. Each rover is also equipped with an array of geological sampling tools including core drills, sample rakes, bulk sample tools, sample bags, and cameras for documentation. The surface hardware is graphically illustrated in Figure 7.

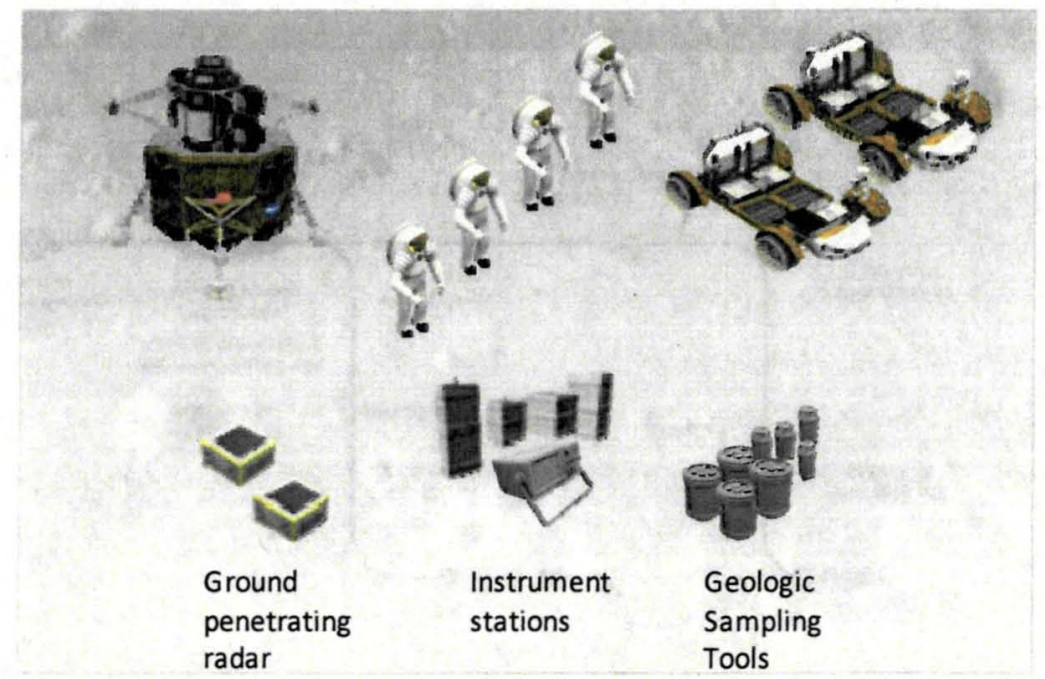

Figure 7. Lunar sortie mission surface hardware elements

\section{Concept of Operations - Tsiolkovsky Crater Sortie}

A detailed day-by-day concept of operations was developed for the Tsiolkovsky crater sortie mission describing all IVA and EVA crew activities. Each surface day involves an EVA by all 4 crewmembers, including rover traverses of between 15 and $32 \mathrm{~km}$. From the landing site near the central peak of Tsiolkovsky crater, unpressurized rover traverses can be performed to the 4 unique geological units within roving distance of the landing site. The geology emphasis of this surface mission will require the crew to stop approximately every kilometer and sample regolith and collect selected rake samples. Along the way the ground penetrating radar will map subsurface structure and determine mare thickness, and the crew will deploy network of four instrument stations. An overview of the surface mission is shown in Figure 8. 


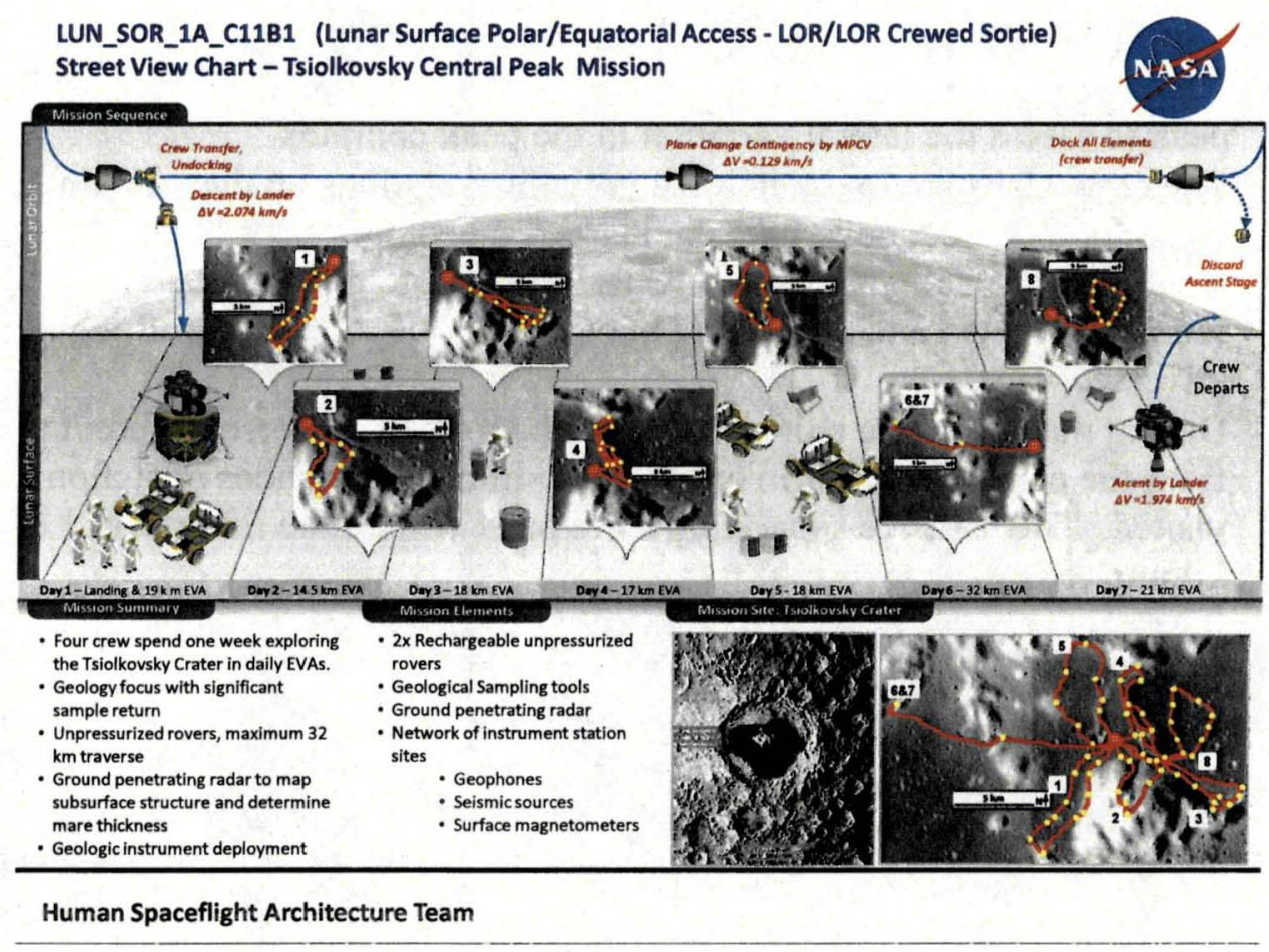

Figure 8. Lunar Sortie Mission Surface Operations "street view"

A typical traverse is shown in Figure 9. The primary objective of this traverse is to characterize the troctolite spur at the east end of the Tsiolkovsky central peak to assess lateral variations of central peak compositions, determine age relationships, and determine the relationships of a peak complex and mare. During the $18 \mathrm{~km}$ traverse, the crew will sample anorthosites, "troctolites", and "norites", observe mineralogic, age and textural relationships, and collect bulk regolith surface samples and rake samples during traverses. In detail, the crew day Concept of Operations is as follows:

- The crew conduct post-sleep activities and prepares the morning meal

- Crew reviews the day's EVA plans with Mission Controllers and check out EVA suits

- The crew don their surface EVA suits via the 4 suit ports on the Habitation Module

- All 4 crewmembers egress the Habitation Module and begin the EVA.

- The crew descends the ladder and detaches the charged rovers from the lander power supply

- The crew loads sample collection equipment required for the EVA onto the rovers

- All 4 crew depart together on 2 unpressurized rovers for the planned 
traverse.

- Crew traverses to the troctolite spur northeast of the Tsiolkovsky central peak to assess the lateral variation in the peak complex

- The crew visits approximately 10 individual stations on the $\sim 18 \mathrm{~km}$ traverse stopping to collect bulk, rake, and targeted geologic samples, and to record geologic observations. The crew performs photo documentation of each sample collected and panoramic photography of each station along the traverse.

- During the EVA, one pair of crewmembers may traverse different paths from the other EVA pair in order to maximize the number of stations visited. The distance separating EVA pairs will not be more than 1 (TBR) kilometer.

- At each station, the crew establishes high data-rate communications with Earth for video and system status monitoring.

- While traversing between stations, the crew has low data-rate communications with Earth.

- Upon return to the lander, the crew attach the rovers to the lander power system for recharge.

- The crew ascends the ladder to the lander's Habitation Module with the samples and imagery collected during the traverse.

- The crew ingress the Habitation Module via 4 suit ports to conclude the EVA.

- The crew transfers the collected samples to the Habitation Module

- The crew recharge PLSS batteries and consumables

- Crew prepares and consumes the evening meal, and transmits the day's traverse imagery to Earth

- The crew configures the Habitation Module for sleep and begins an 8-hour sleep period. 


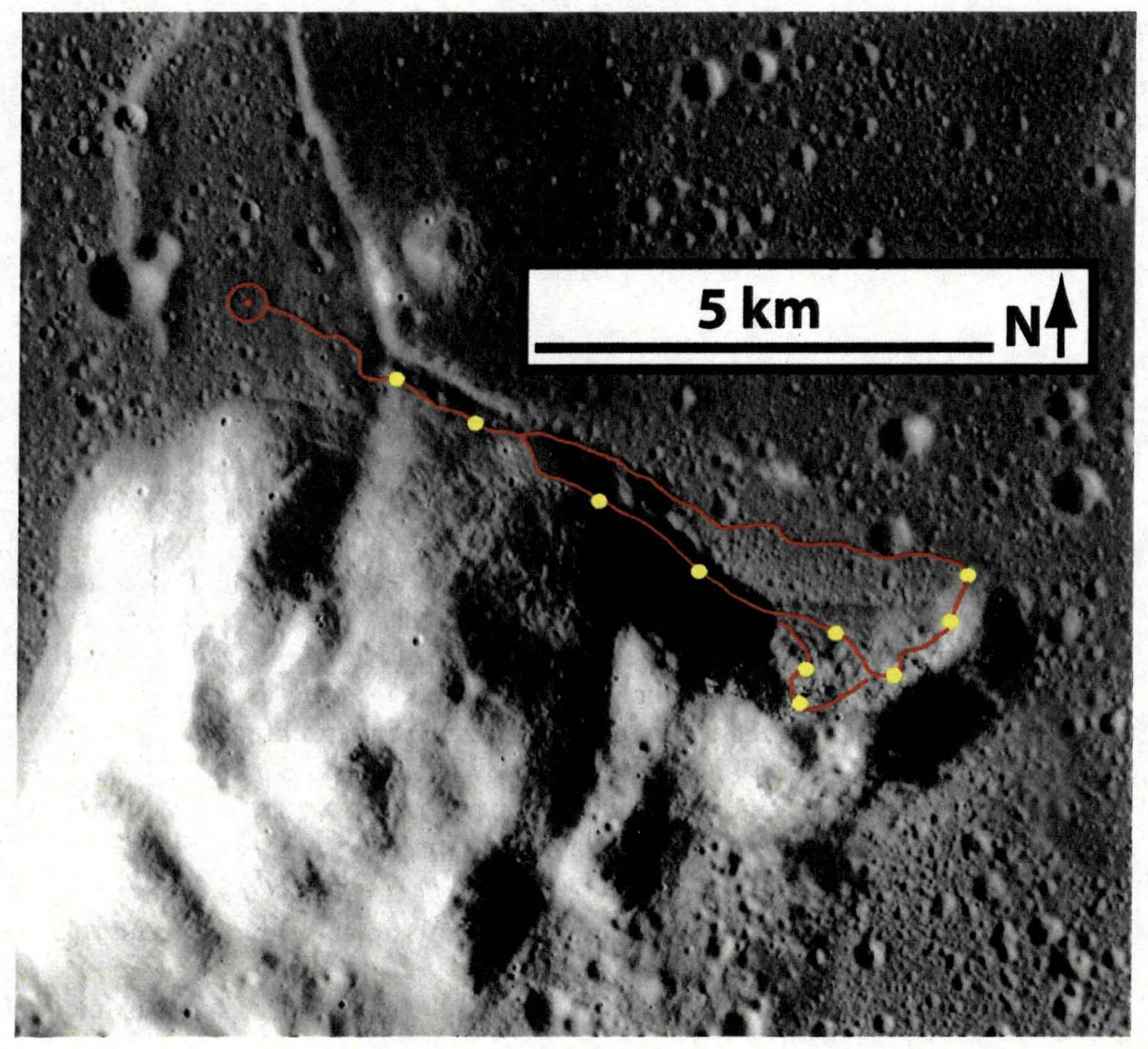

Figure 9. Typical sortie mission traverse path with scientific stations shown

\section{Lunar Sortie Mission Observations}

Working the details of each surface reference mission gives NASA engineers the opportunity to identify areas for improvement, and to identify inconsistencies with the matching transportation DRMs. The lunar geological sortie presented above is a relatively simple mission, yet by detailing the concept of operations, a number of issues were identified. A prime example is the need for additional delivered payload mass - the $500 \mathrm{~kg}$ delivered down-mass is insufficient for delivery of 2 unpressurized rovers and science instruments for an aggressive 7-day geological sortie, which requires on the order of $700 \mathrm{~kg}$ of payload. The lengthy traverses also require both unpressurized rovers (and all 4 crewmembers) to remain together, since a single unpressurized rover will limit traverse radius to walk back distances $(\sim 10 \mathrm{~km})$. Certain traverses split the crew midway to maximize science sites visited, but limit the separation distances to less than $1 \mathrm{~km}$. 
Reviewing the crew timeline, 7 consecutive 4-crew EVAs will challenge the crew's physical limits, especially on landing and departure day, where EVAs and significant mission flight events will occur back-to-back. This may exceed a reasonable crew day timeline on those days. The 8 hour EVAs will also challenge the crew's meal schedule, necessitating a 2 meals per day schedule - post sleep/prior to EVA, and pre-sleep/post-EVA. Finally. transferring the daily collected samples into the Pressurized Volume will produce a challenge to control dust and sample environment integrity.

\section{EXTENDED STAY HUMAN LUNAR MISSION}

An Extended Stay mission makes use of pre-deployed surface assets to extend the crewed surface stay beyond seven days. During the Cycle $\mathrm{C}$ excursion phases (polar only), these extended stay missions do not have large habitats to enable stays longer than 28 days. The crew will live in the small pressurized rovers known as Space Exploration Vehicles (SEV) which also provide mobility for excursions lasting up to 28 days depending on the available logistics supplies. The lander for these missions will not include an airlock. Crew egress and ingress from the ascent module is achieved through suit ports. SEV's with Portable Utility Pallets (PUP) will provide all habitation and power during these missions. The HAT has analyzed an extended stay human lunar mission with a 28 day duration at either lunar pole. The ground rules and assumptions are:

- Multiple (5) extended stay (up to 28 day) missions, beginning with robotic precursors and initial cargo landers

- Lunar surface emphasis is to test the capabilities and learn self-sufficiency in preparation for human Mars missions

- 4 crew

- Polar site

- Small cargo landers (1 mt)

- Larger cargo landers (8 $\mathrm{mt}$ )

- Automated pre-deployment

- Rover chassis

- Resources

- Pressurized Rover: Mobile Habitation

- Long-distance mobility (100's km)

- Technologies:

○ Mobility

- Dust control

- Habitation

- Autonomous landing and hazard avoidance

- Advanced surface power (if available) 


\section{Transportation Architecture}

The HAT Cycle $\mathrm{C}$ transportation consists of a heavy lift launch vehicle that can deliver cargo or crew. Some cargo and robotic precursor missions are predeployed (with the help of a cryogenic propellant stage (CPS) for lunar orbit insertion), and the crew in a Multi-Purpose Crew Vehicle (MPCV) capsule meets the lunar lander in a lunar orbit rendezvous (LOR). After the surface mission the crew departs in an ascent stage and does another LOR with the MPCV that has remained in orbit. The journey home is completed in the MPCV with a direct entry. International launchers may be used for smaller cargo landers and pre-cursor robotic missions.

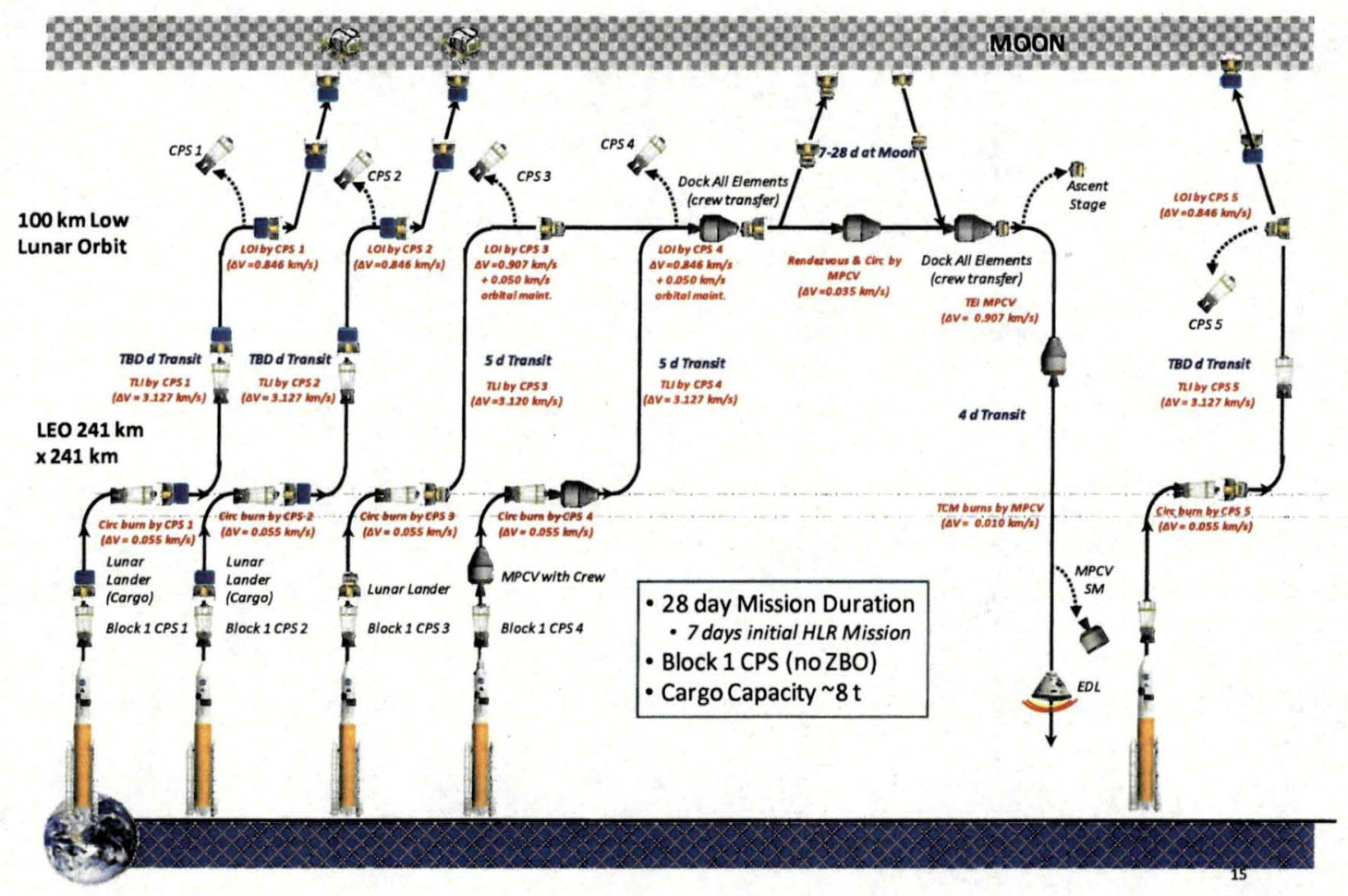

Figure 10: HAT Cycle C Lunar Transportation Architecture (U.S. Launcher Only)

\section{Lunar Surface Activities}

The lunar extended stay exploration begins with a small $1 \mathrm{t}$ class lander delivering several small robots to one of the lunar poles. These robots work together to identify a suitable landing site for an upcoming $8 \mathrm{t}$ human class lander. In addition to local reconnaissance, the small rovers gather science data, validate technologies and refine concurrent operations. This will be the first time ever that multiple robots will be working together in close proximity on another celestial body. The robots will practice servicing operations, scout the region for future crew/cargo landing areas, and deploy 
landing aides. All robots will send back to Earth a steady stream of engaging and informative data and video, including the descent and touchdown of future crewed/cargo landers. A crewed lunar flyby is performed, utilizing for the first time the cryogenic propulsion stage to leave the Earth's gravity well. A year later, an un-crewed human scale $8 \mathrm{t}$ lander touches down at the site identified by the small robots. It is carrying a version of the mobility chassis used by the crewed small pressurized rover. It could also carry small communications relay satellites that are deployed in lunar orbit to enable better coverage of the poles before descending. The mobility chassis will operate in autonomous and ground supervised modes at speeds and ranges far exceeding any previous planetary surface rover, and is outfitted with enough energy storage to survive up to 14 day eclipse periods. It will also be outfitted with hundreds of kilograms of science instruments and manipulators. The vast science payload, substantially increased speed and range along with the capability to survive lunar eclipse will allow it to traverse long distances away from the polar landing site to achieve regional exploration. In addition to its own science payloads, it will also be capable of transporting one or more of the previously delivered small robots as it explores which could be used for servicing and remote observation. This mobility platform will provide multiple partnering opportunities while substantially reducing risk for future exploration missions.

The lunar surface roving capability is augmented for a few years during this period by crewed missions to low lunar orbit that are testing the transportations system as well as refining techniques for tele-operating the surface assets from low lunar orbit. This activity is analogous to operating rovers on the surface of Mars from Mars orbit, as eventually may occur during a Mars orbital mission. An opportunity during this period occurs for practicing lunar orbit rendezvous techniques with crew rated systems (such as ISS technology module, alternate transportation systems, SEV/Ascent module prototypes). Extended tele-operations supported by the communication relays can be achieved by docking to these crew rated systems for periods beyond nominal crew capsule lifetimes (typically about 7-9 days in LLO). Docking to systems with additional habitable volume will allow for longer duration stays in low lunar orbit.

The next segment of the lunar exploration phase begins a few years later. The robotic precursor work has incrementally built up confidence in operations and systems design in preparation for more aggressive lunar exploration with humans. Human Lunar Return (HLR) occurs at one of the lunar poles due to the favorable solar and thermal conditions, preventing exposure of the systems to the harshest operational environment of a full, approximately 15 day, lunar night.

After the site on the moon that will host HLR has been sufficiently investigated by the robots, the deployment of the exploration infrastructure begins. The deployment of the pressurized rover and the crewed ascent module occur within a year of each other, thus increasing the potential to share systems development like Environmental Control and Life Support Systems (ECLSS), thermal and power. Several years after the initial robotic missions, but before the first crewed mission, a small pressurized rover 
with supporting power infrastructure lands in the polar region and self-deploys. The surface arrival is directed by the robotically emplaced landing aides.

The small pressurized rover is initially tested, then sent on excursions (in a ground supervised mode) progressively further away from the landing location, beyond the range of the small robots, to identify opportunities and optimal paths that can be used by the humans on the first crewed mission. A year later, when the humans along with any critical spares arrive, the fully checked out rovers (original mobility chassis and the new small pressurized rover) are waiting for them. The crew then performs up to a 14 day mission (seven days initially planned), exploring the near polar region and practicing operations and contingency scenarios for upcoming traverses. Having two human scale rovers (one pressurized, one unpressurized) offers redundancy and rescue capabilities in the event one rover becomes non-operational. The crew leaves the surface at the end of their mission while the robots continue exploring before the next crew arrives, enhanced by PUPs and cargo delivered by small international landers. Six months later another small pressurized rover is delivered, autonomously deployed and tested, so that it can join the previously delivered mobility chassis and pressurized rover at the next crewed landing location.

The next crew arrives six months later and performs a 14 day mission using the extended range and duration resulting from coupling the small pressurized rovers to the PUPs. A crew does not return to this location for a year as the small pressurized rovers, the servicing robots and the PUPs perform extensive ground supervised exploration. One year after HLR, a third crewed mission arrives at the pole, with the goal of lengthening the mission duration to 28 days. This cycle repeats for two more years, with each mission lasting 28 days, enabled by the mobile infrastructure meeting the crew at new polar region landing sites and delivery of logistics and science instruments by small $1 \mathrm{t}$ landers. By the time the mobile infrastructure is near the end of its design life, humans have spent 105 days on the lunar surface exploring and tested key planetary surface capabilities and operations.

\section{Elements Required}

The elements required to complete a 28 day extended stay are articulated in Table 1. 


\begin{tabular}{|c|c|c|}
\hline Element & QTY & Notes \\
\hline Crew & 4 & International Astronaut Crew \\
\hline $\begin{array}{l}\text { PUP (Portable Utility } \\
\text { Pallet) }\end{array}$ & 3 & $\begin{array}{l}100 \mathrm{~kW} \text {-hr battery storage each } \\
2 \mathrm{~kW} \text { solar array each } \\
\text { Transported by SEVs }\end{array}$ \\
\hline $\begin{array}{l}\text { PCT (Portable } \\
\text { Communications Terminal) }\end{array}$ & 1 & $\begin{array}{l}\text { Provides high bandwidth communications } \\
\text { Transported by PUP }\end{array}$ \\
\hline Robotic Precursor 1 (R1) & 1 & Small International Science Rover \\
\hline Robotic Precursor 3 (R3) & 1 & Small International Science Rover \\
\hline $\begin{array}{l}\text { UPR (Unpressurized } \\
\text { Rover) }\end{array}$ & 1 & $\begin{array}{l}\text { Provides Excursion Capability before } \\
\text { second SEV arrives }\end{array}$ \\
\hline $\begin{array}{l}\text { Off-loader (LSMS or } \\
\text { Cradle) }\end{array}$ & 1 & $\begin{array}{l}\text { Can tele-robotically offload cargo landers } \\
\text { or be used off the back of an SEV. }\end{array}$ \\
\hline Science Package & 1 & Pre-deployed in second mission \\
\hline Logistics & 9 & $\begin{array}{l}\text { Multiple logistics payloads required for } 28 \\
\text { day capability }\end{array}$ \\
\hline $\begin{array}{l}\text { STM (Suit port Transfer } \\
\text { Module) }\end{array}$ & 1 & $\begin{array}{l}\text { Allows transfer of material through a Suit- } \\
\text { port }\end{array}$ \\
\hline $\begin{array}{l}\text { SEV (Space Exploration } \\
\text { Vehicle) }\end{array}$ & 2 & $\begin{array}{l}200 \mathrm{~kW} \text {-hr battery storage each } \\
\text { Average speed toward destination }=5 \\
\mathrm{~km} / \mathrm{hr}\end{array}$ \\
\hline Robotic Precursor 2 (R2) & 1 & $\begin{array}{l}\text { Small NASA Robotic Assistant \& Science } \\
\text { Rover }\end{array}$ \\
\hline $\begin{array}{l}\text { ALC (Airlock Logistics } \\
\text { Carrier) }\end{array}$ & 7 & Pressurized Logistics \\
\hline
\end{tabular}

Table 1: Lunar Surface Elements for a 28 Day Extended Stay DRM 


\section{Manifest of Missions}

The sequence of missions and elements or manifest can be graphically shown in a "street view" picture as demonstrated below. Four large cargo landers, six small cargo landers, result in five crewed missions for this HAT Cycle C DRM. A notional location of landing at the South Pole Shackleton crater rim is shown but there are a number of other candidate locations as well, which are interesting from a science and exploration point of view.

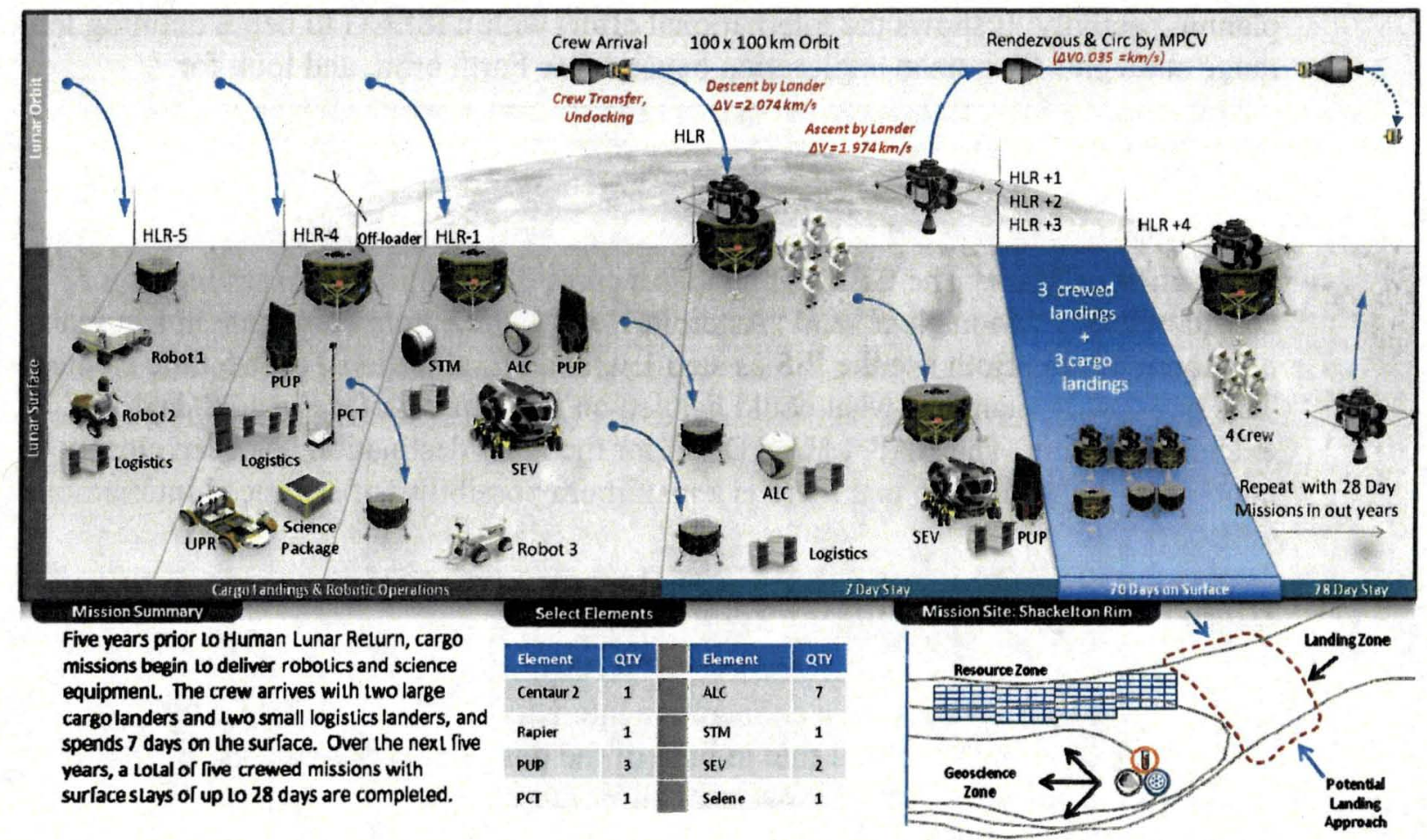

Figure 11: "Street View" of a 28 Day Lunar Extended Stay Mission

\section{Extended Stay DRM Observations}

This DRM has identified a preliminary set of elements, concept of operations and a launch and deployment manifest that seems to be feasible. However, there are many details regarding logistics and habitation that have not been addressed which warrant further study. It is not clear that 4 crew can live productively in 2 SEV's for 28 days without some new strategies such as caching supplies on the lunar surface for periodic replenishment. Suit ports are assumed in this study although they have never been used in space before. Advanced energy storage will be required or the SEV will have to stay in the sunlit regions to use photo-voltaic power. In addition, risk reduction through the use of robotic precursors is highly desirable but would require significant international coordination for transportation, deployment and operation with today's planned U.S. launchers. 
This study is considered a point of departure for discussion and consideration but will require further analysis to be considered complete.

\section{GLOBAL EXPLORATION ROADMAP LUNAR MISSION}

The Global Exploration Roadmap (GER) is a tool developed by ISECG participating agencies to facilitate advancement of coordinated and cooperative human space Exploration plans and programs, building on the vision in the Global Exploration Strategy. The GER reflects the start of an international human space exploration planning activity. It shows the international effort within ISECG to begin defining long range strategies for human exploration beyond low Earth orbit, and look for opportunities to coordinate and cooperate in near-term activities which prepare for these challenging missions.

The ultimate goal that has been agreed on by the ISECG is the human exploration of Mars. The GER roadmap has currently identified two strategies for consideration: "Moon Next" and "Asteroid Next", which both culminate in a human mission to Mars. Both use the ISS as step 1 with an Exploration Test Module element. This paper only examines what could happen on the Lunar surface in a 28 day extended mission. The NASA HAT DRM for the lunar destination has been closely coupled with the GER so that there is a maximum possibility of enhanced international collaboration.

\section{Global Exploration Roadmap Identified Cases}

The Global Exploration Roadmap (GER) provides a detailed architectural reference mission design with a cooperative international focus. Not surprisingly, lunar surface missions play a central role in both of the possible exploration paths articulated. The GER derived architecture showcases an increase in surface stay time that evolves over time and provides optional increased capability in the form of ISRU to each branch. In the end, seven different branches in the lunar trade tree can be tied to a GER framework. Figure 11 outlines the branches that align with a base-level GER mission as well as the potential off-shoot alternatives. 


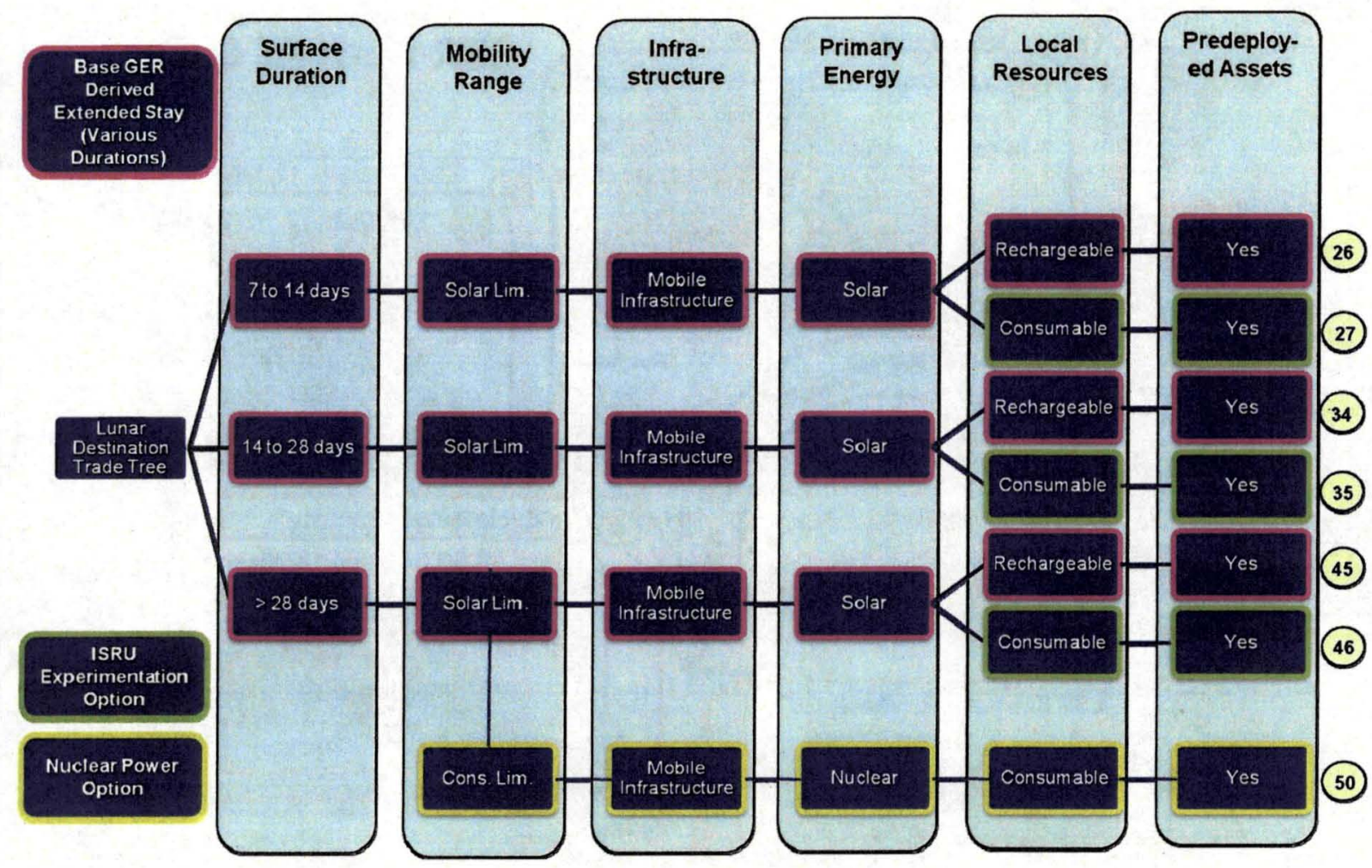

Figure 12: Multi-duration, Multi-path GER-derived Missions on the Lunar Trade Tree

The mini street view diagrams for the GER-derived missions are given in 12 . Each mission is identical in capability aside from the increase in stay duration from chart to chart. Also, each mission pair presents ISRU for consumable capability (small production quantities) as a mission enhancer. The final mission scenario adds nuclear energy as an option.

Note that 5 out of 7 of the mission scenarios can be tied to the Constellation driven Lunar Surface Study (LSS) at NASA. This demonstrates close alignment between the international efforts and NASA's own in-house work. 


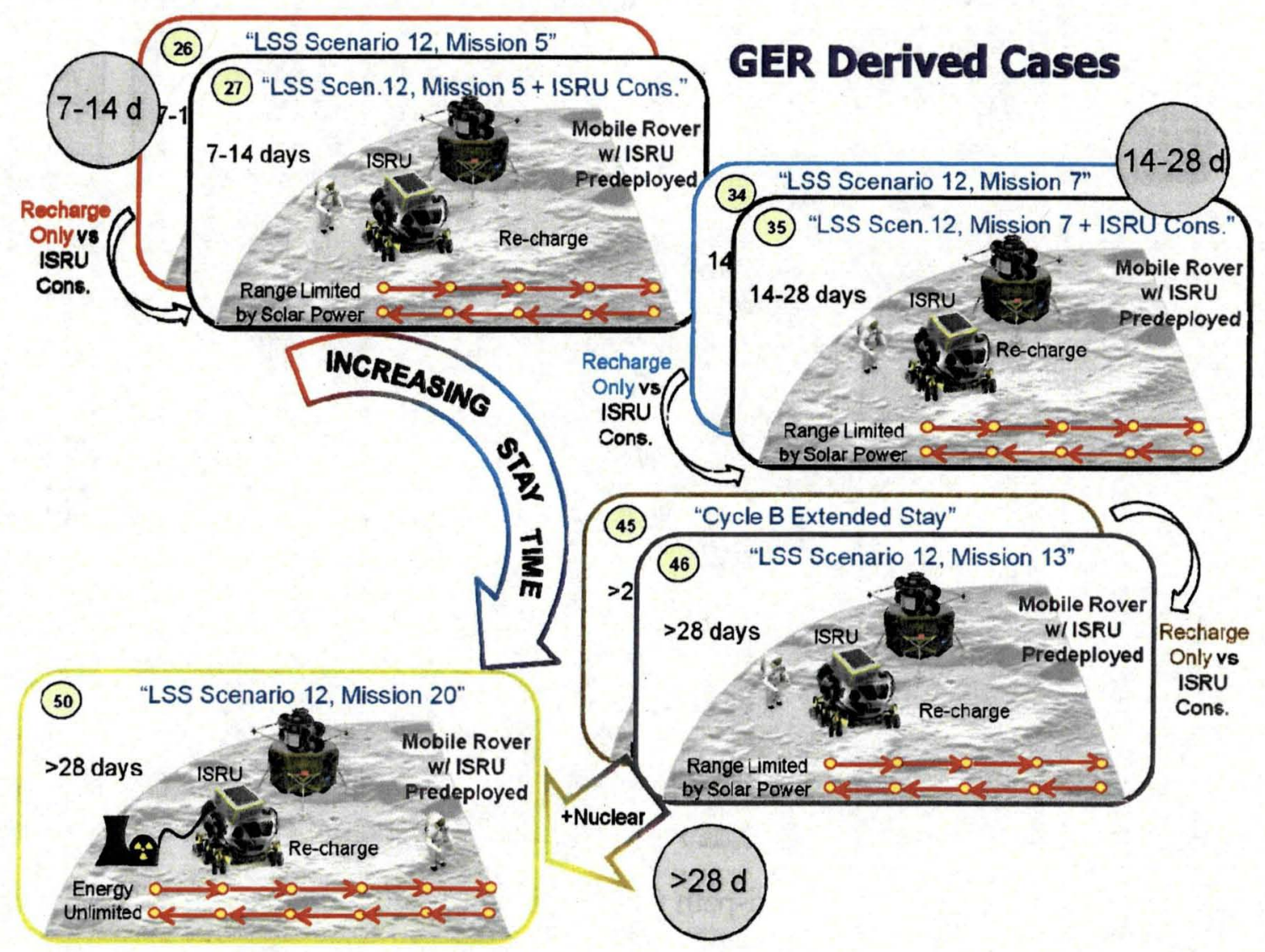

Figure 13: "Street View" Diagrams for GER-derived Mission Options

\section{CONCLUSION}

The HAT lunar destination team has defined a continuum of surface reference missions ranging from simple sortie missions to fully capable international extendedstay missions. Each mission represents a logical path in a comprehensive decision tree of lunar surface design options. Representative surface DRMs have been worked in detail to define operations concepts, timelines, hardware elements and issues. The HAT team will continue to explore other paths through its trade space in order to identify destination missions that balance performance and affordability. 


\section{REFERENCES}

Augustine et al. (2009). "SEEKING A HUMAN SPACEFLIGHT PROGRAM WORTHY OF A GREAT NATION", Review of US Human Spaceflight Committee Report, White House Office of Science and Technology Policy, Washington, D.C.

International Space Exploration Coordination Group (ISECG) (2011)." The Global Exploration Roadmap", http://www.globalspaceexploration.org/web/isecg/documents. 


\section{NASA Human Spaceflight Architecture Team (HAT) Lunar Destination Activities}

Rob Mueller John Connolly Ryan Whitley
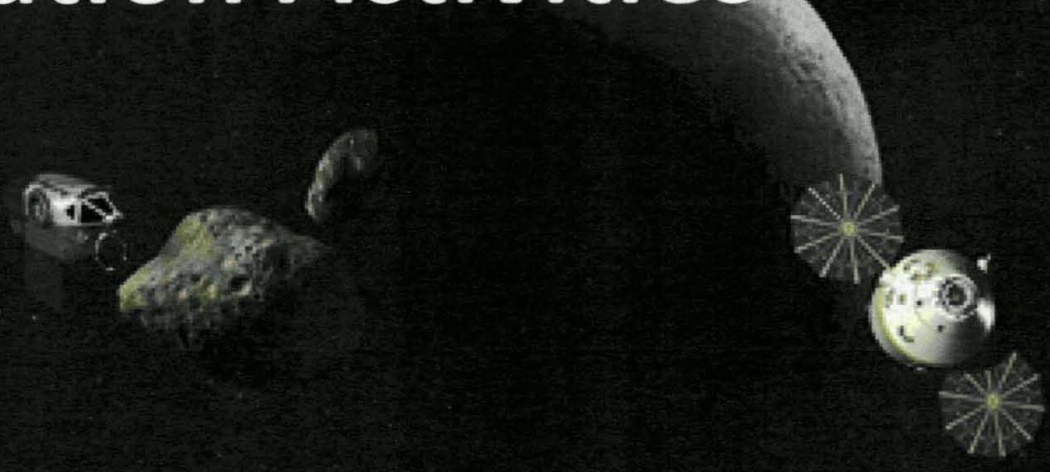

April 18, 2012 
Lunar Destination Trade Tree

$\checkmark$ GER-Derived Lunar Destination DRM 


\section{Full Lunar Trade Tree 1440 Branches}

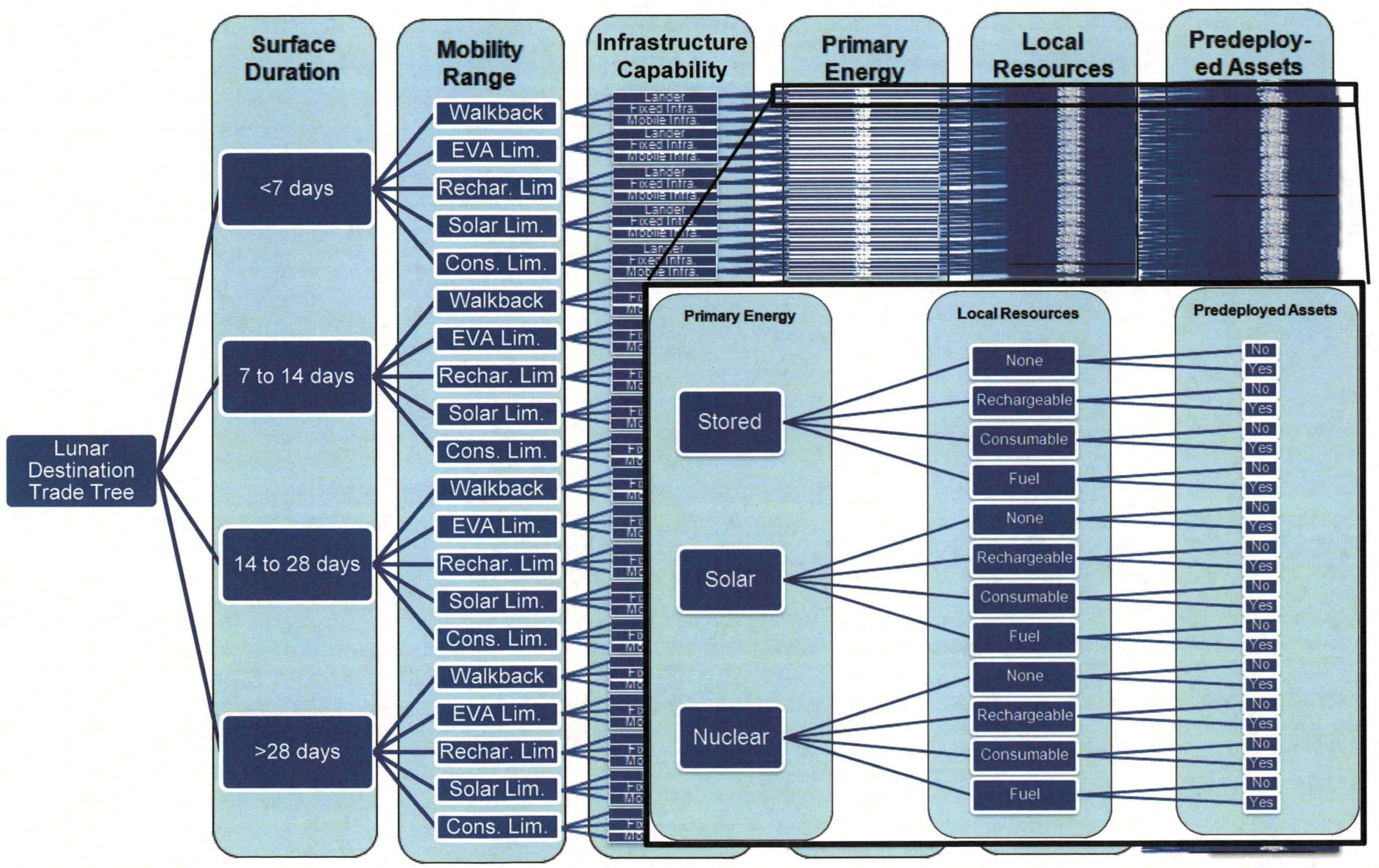




\section{Full Lunar Trade Tree 1440 Branches}

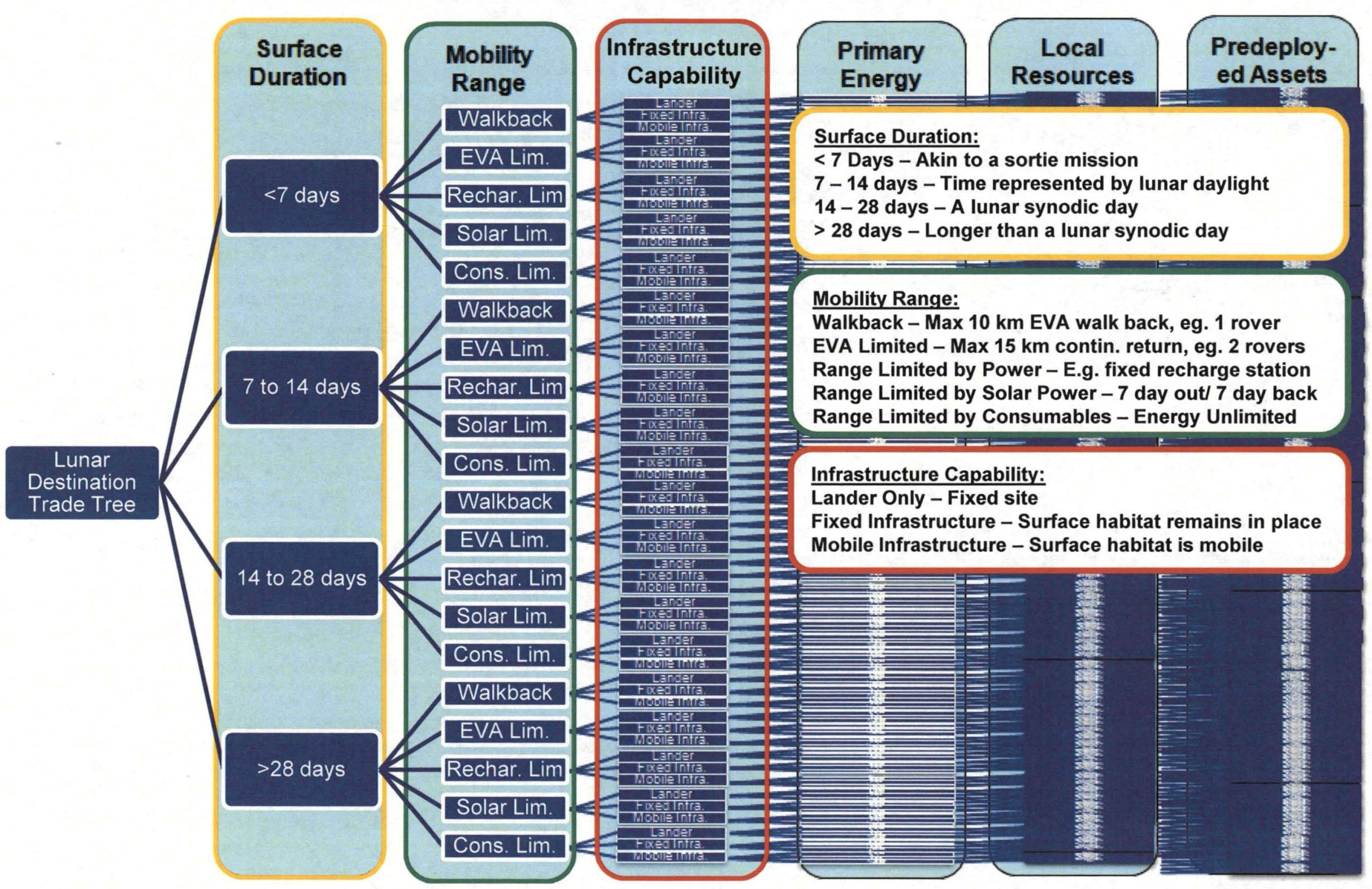




\section{Zoomed Lunar Trade Tree}

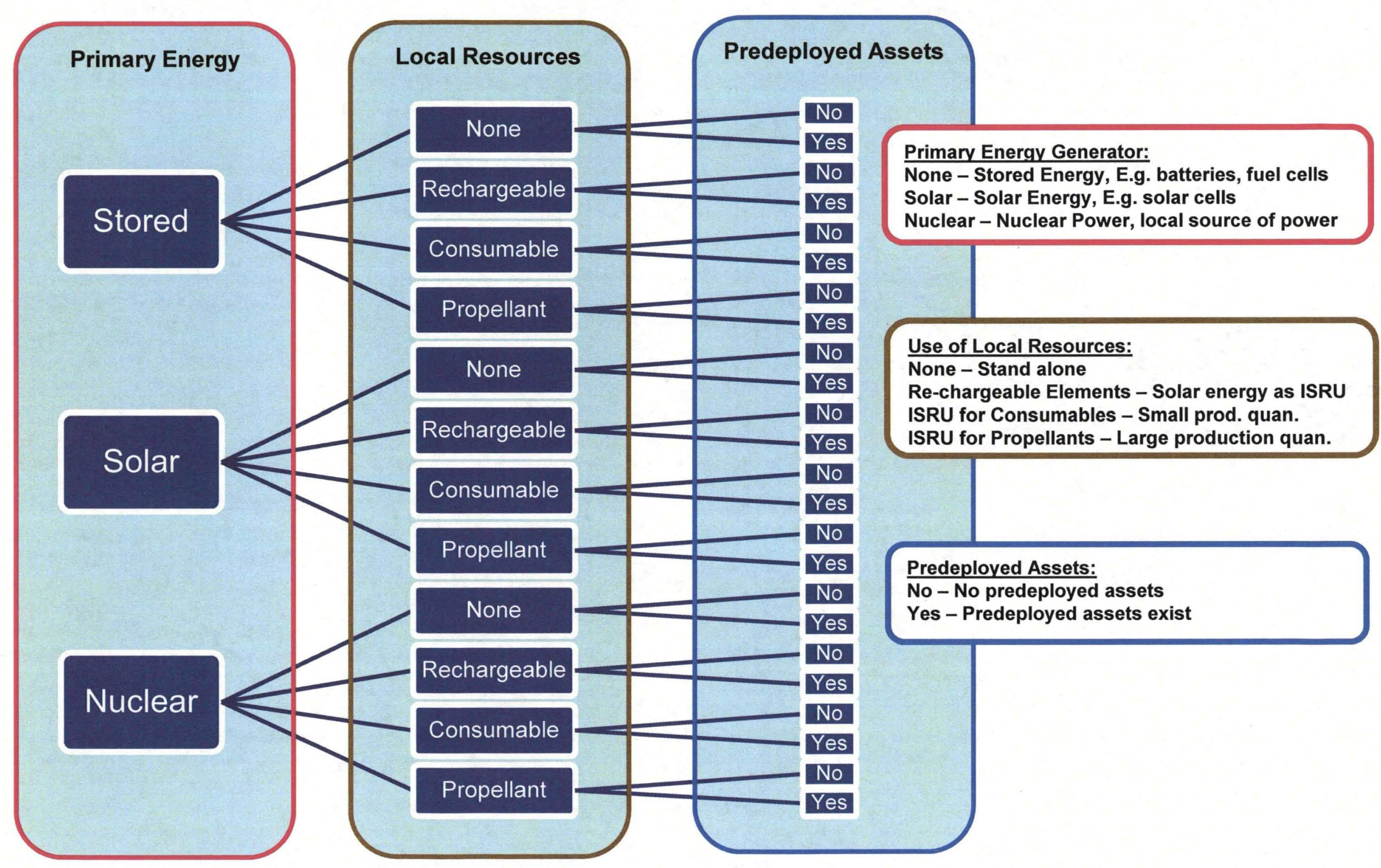




\section{Lunar Trade Tree: Logical Disconnect Branch Trimming}

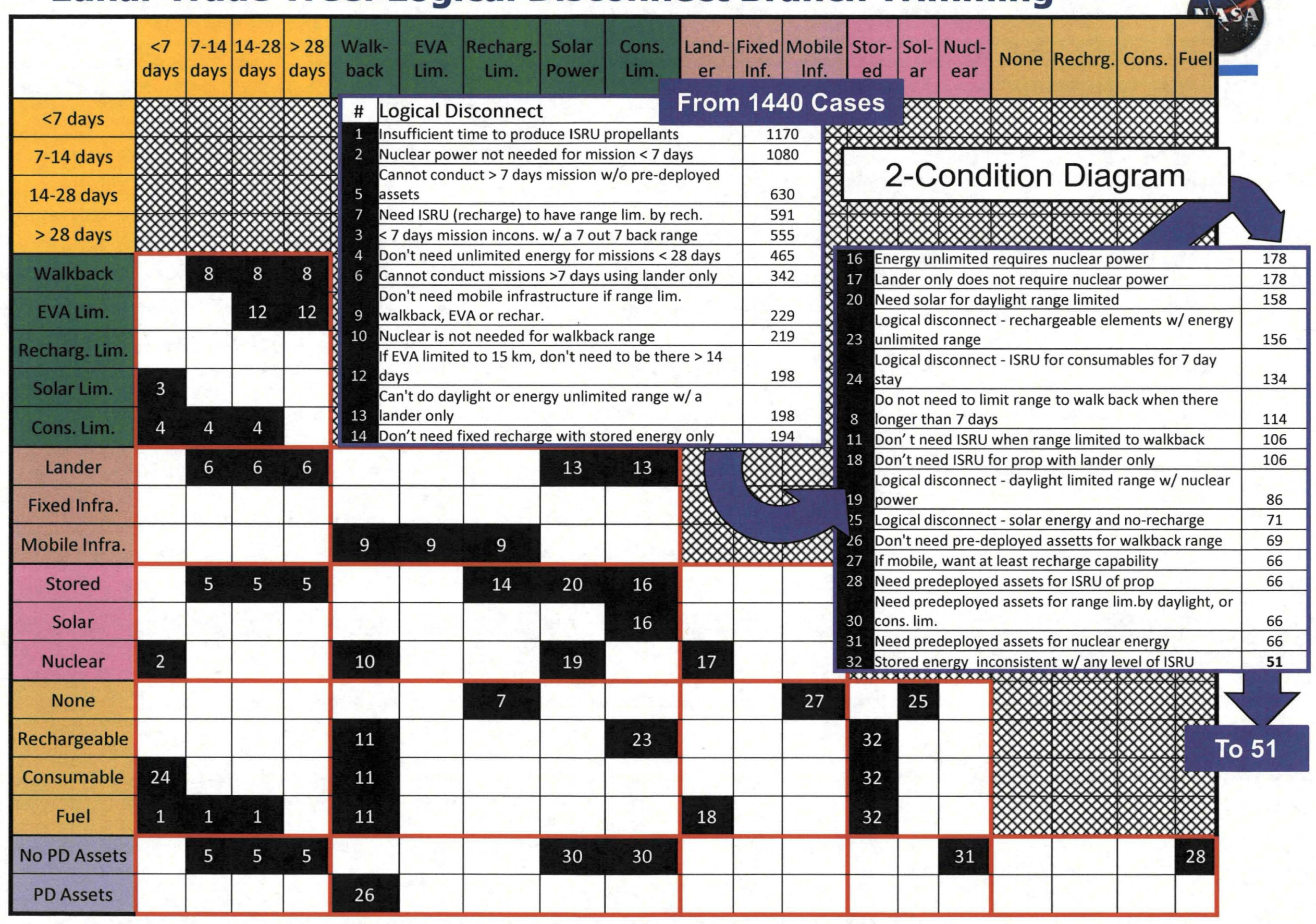




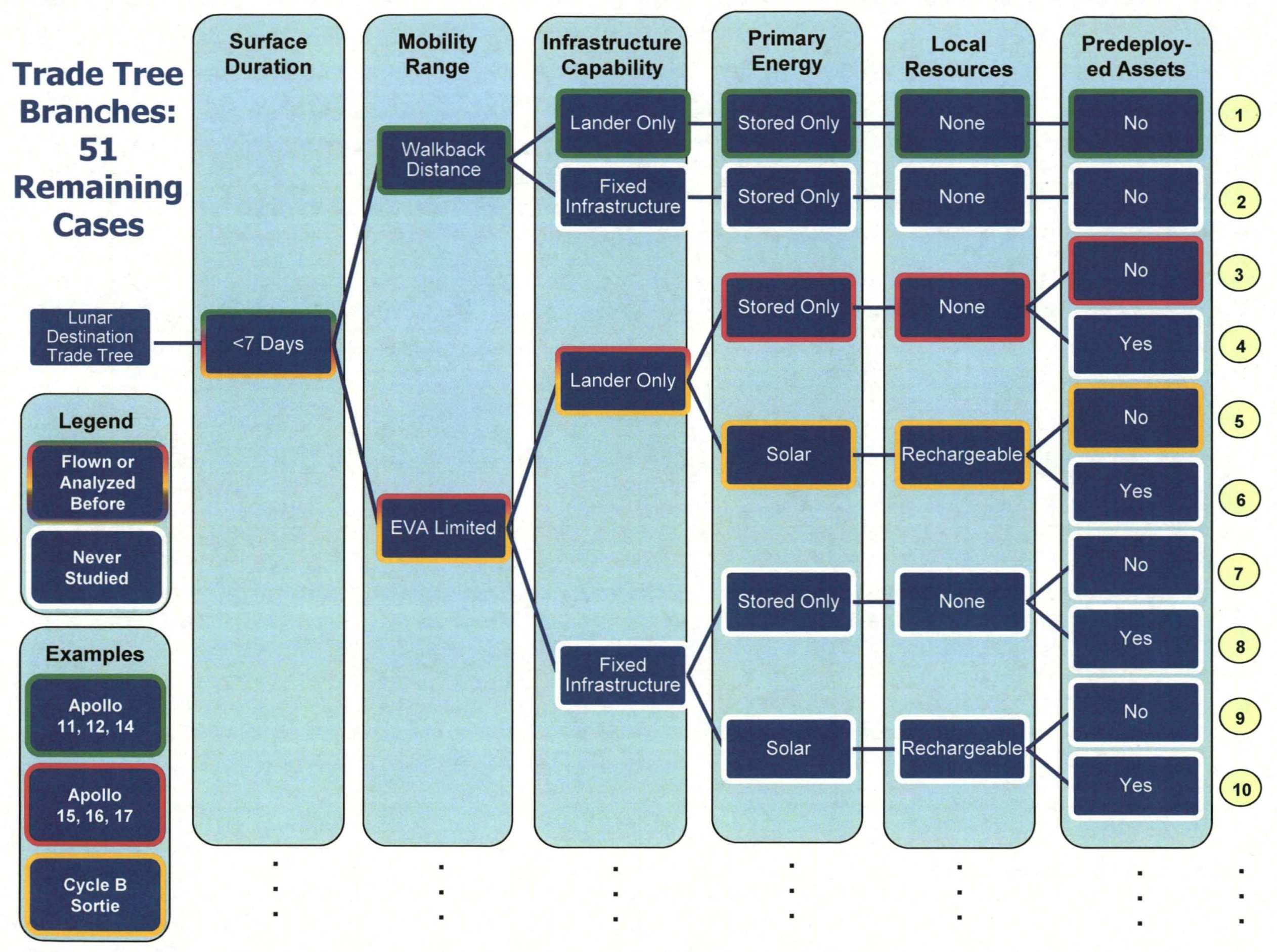




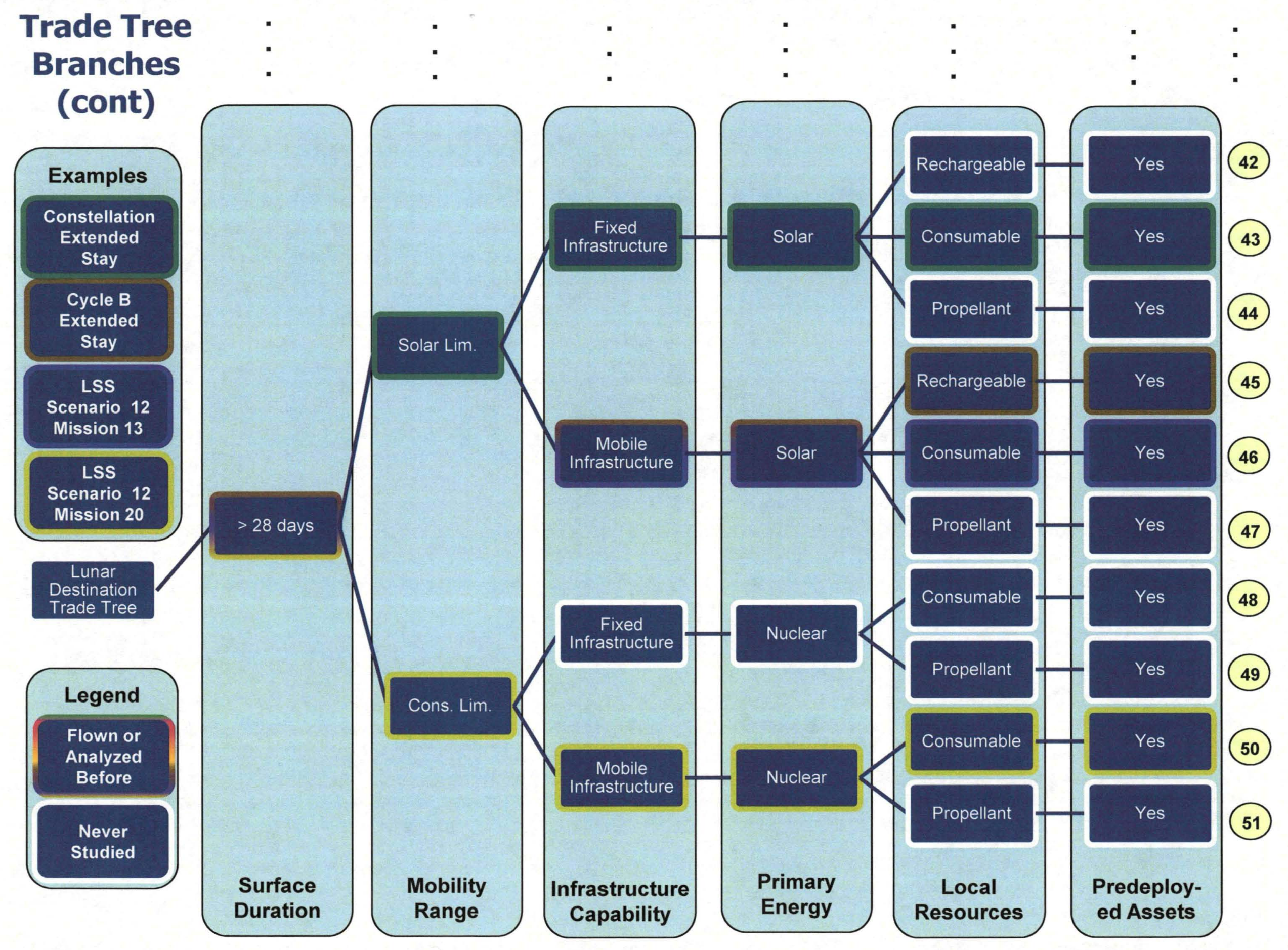




\section{Analyzing Remaining Cases}
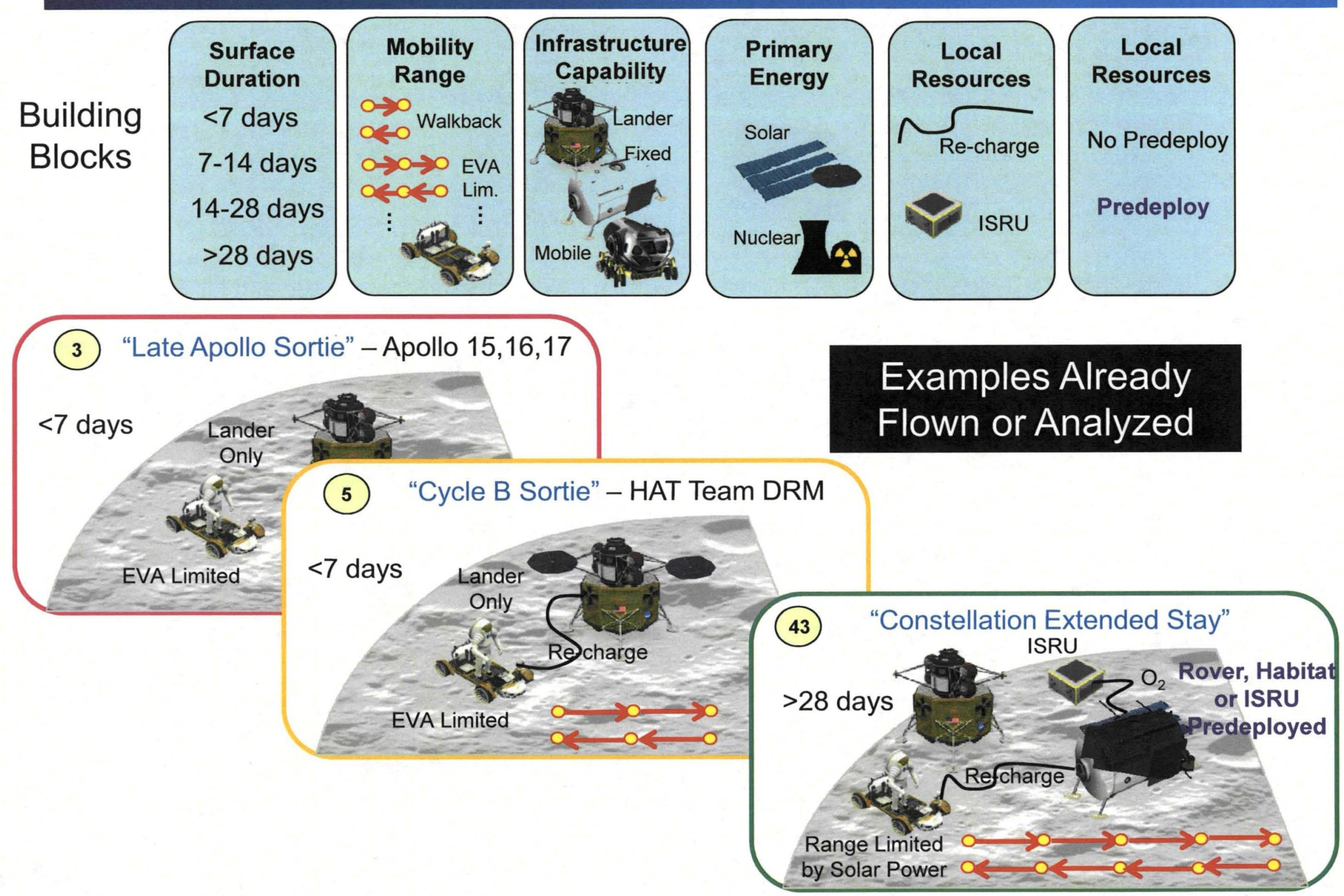


\section{Analyzing Remaining Cases}

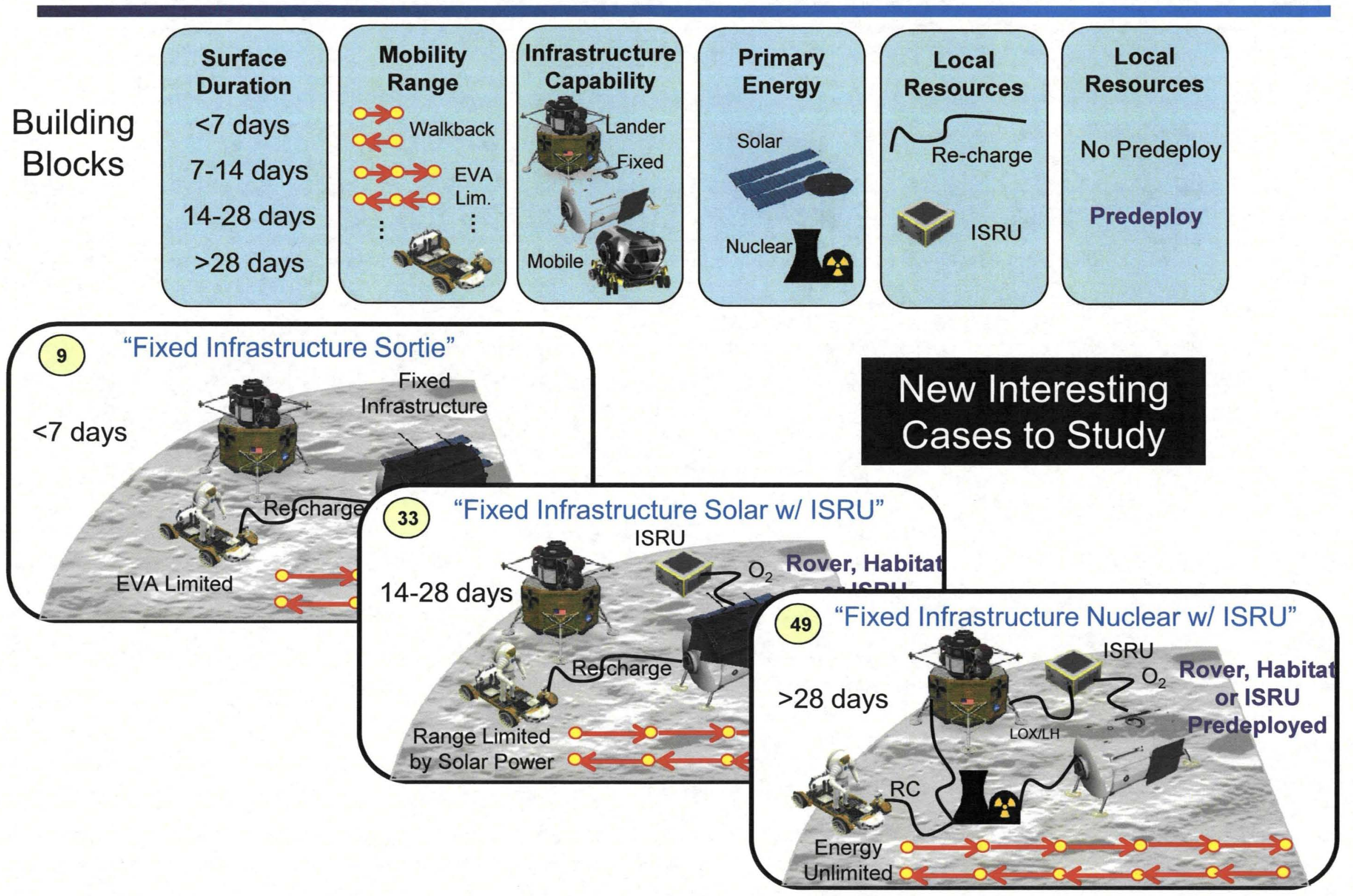




\section{Multiple Paths for GER Derived}

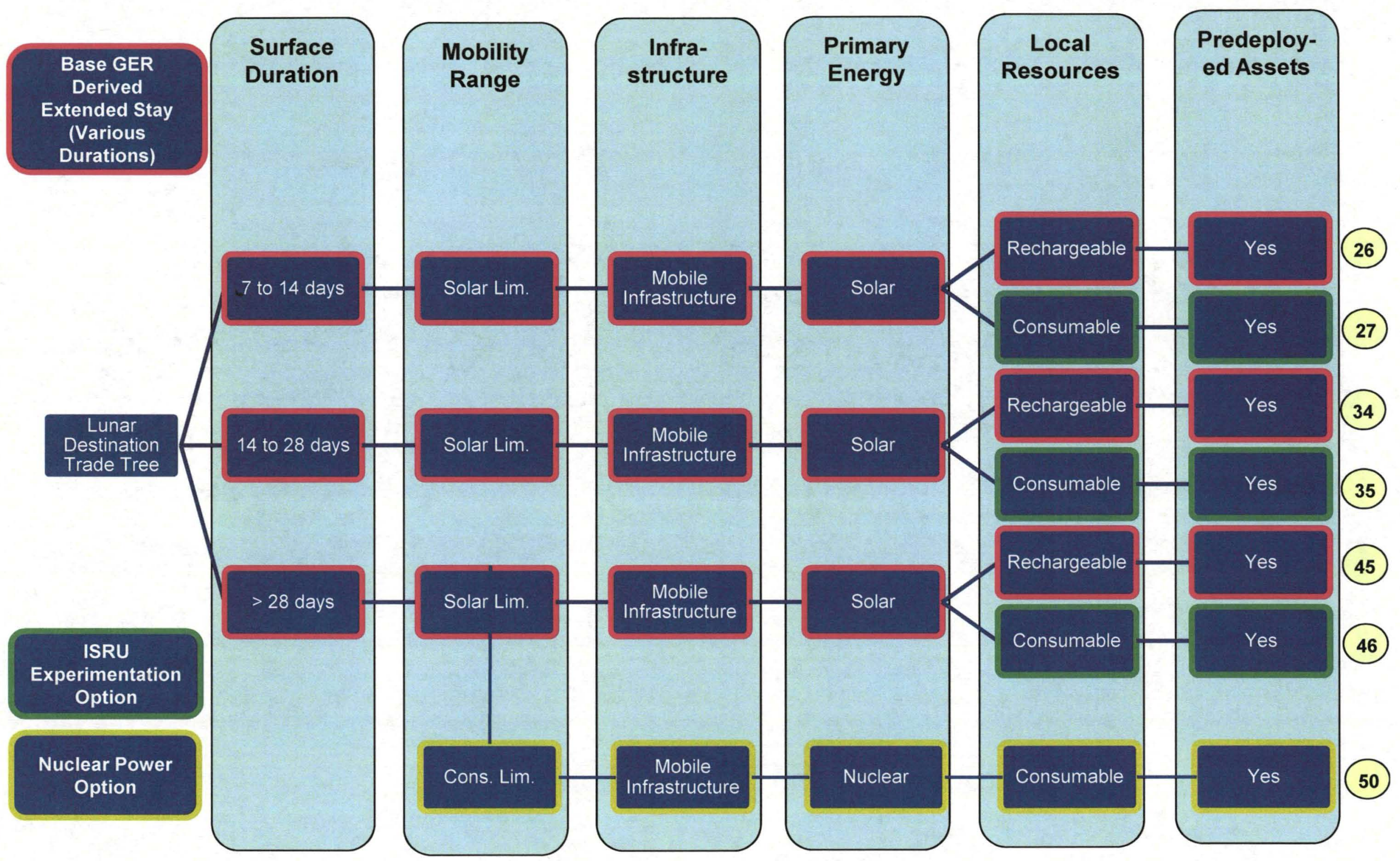




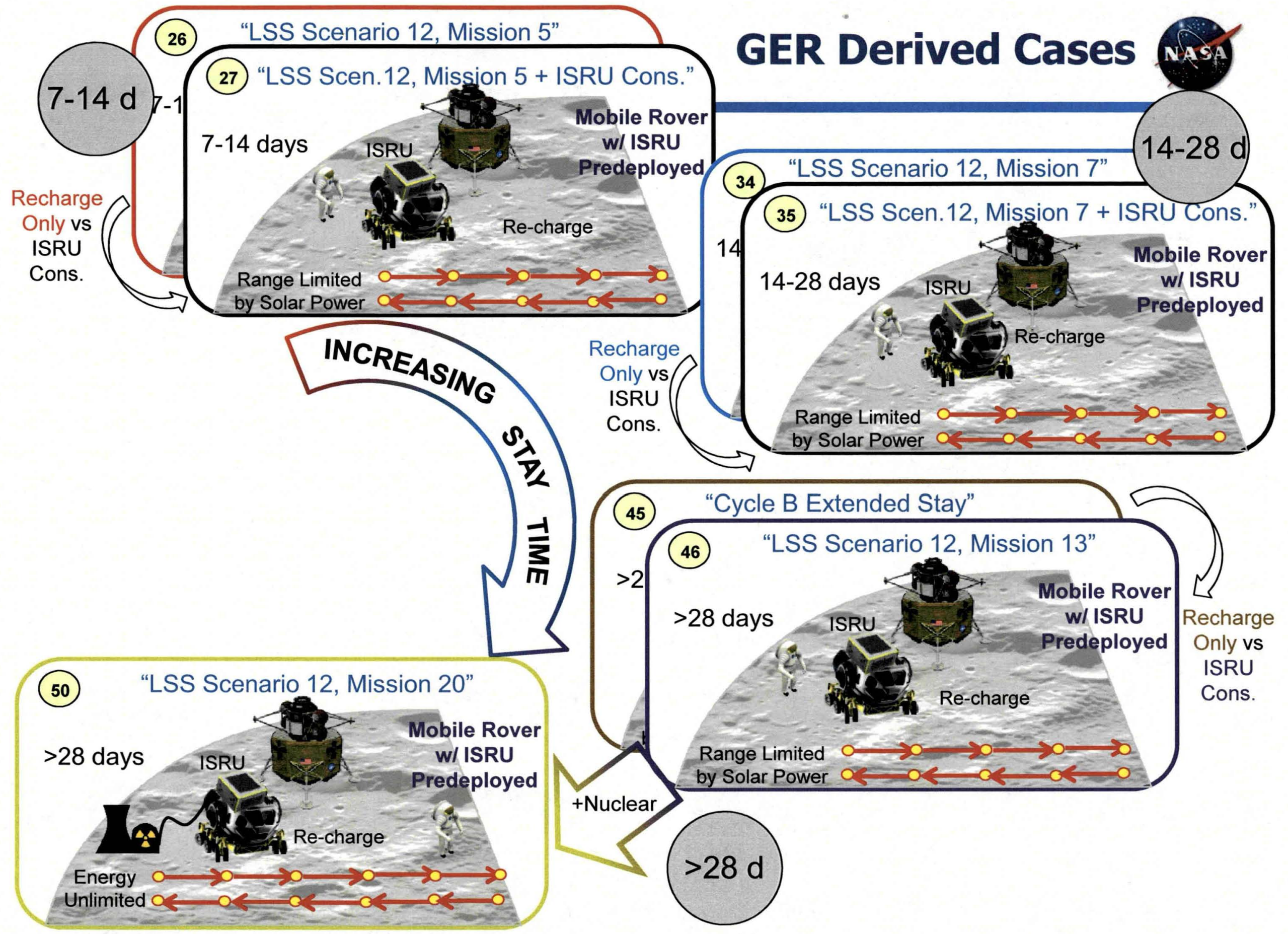




\section{GER-Derived Lunar Destination DRM}

\section{This lunar destination DRM is derived from the GER Lunar mission:}

Multiple (5) extended stay (up to 28 day) missions, beginning with robotic precursors and initial cargo landers

- Lunar surface emphasis is to test the capabilities and learn self-sufficiency in preparation for human Mars missions

- 4 crew

- Polar site

- Small cargo landers (1 mt)

- Larger cargo landers (8 mt)

- Automated predeployment

- Rover chassis

- Resources

- Pressurized Rover: Mobile Habitation

Long-distance mobility (100's km)

Technologies:

- Mobility

- Dust control

- Habitation

- Autonomous landing and hazard avoidance

- Advanced surface power (if available) 
Early focus on advancing the capabilities needed for planetary surface exploration

- Mars-forward technology needs

- Extreme surface mobility

- Surface Power

- Operations in a dusty environment

- Precursor robotic missions

- To maximize the time (efficiency) of human explorers by preparation

- To advance scientific knowledge

- To refine surface operation techniques

- Mobile Infrastructure with Multiple Landing Sites

- Robotic Activities while humans are not there

- Mars surface exploration risk-reduction activities

Evolutionary testing strategy 


\section{HAT Cycle C /GER - Lunar Surface Mission 7-28 day Extended Stay Mission with HLLV}

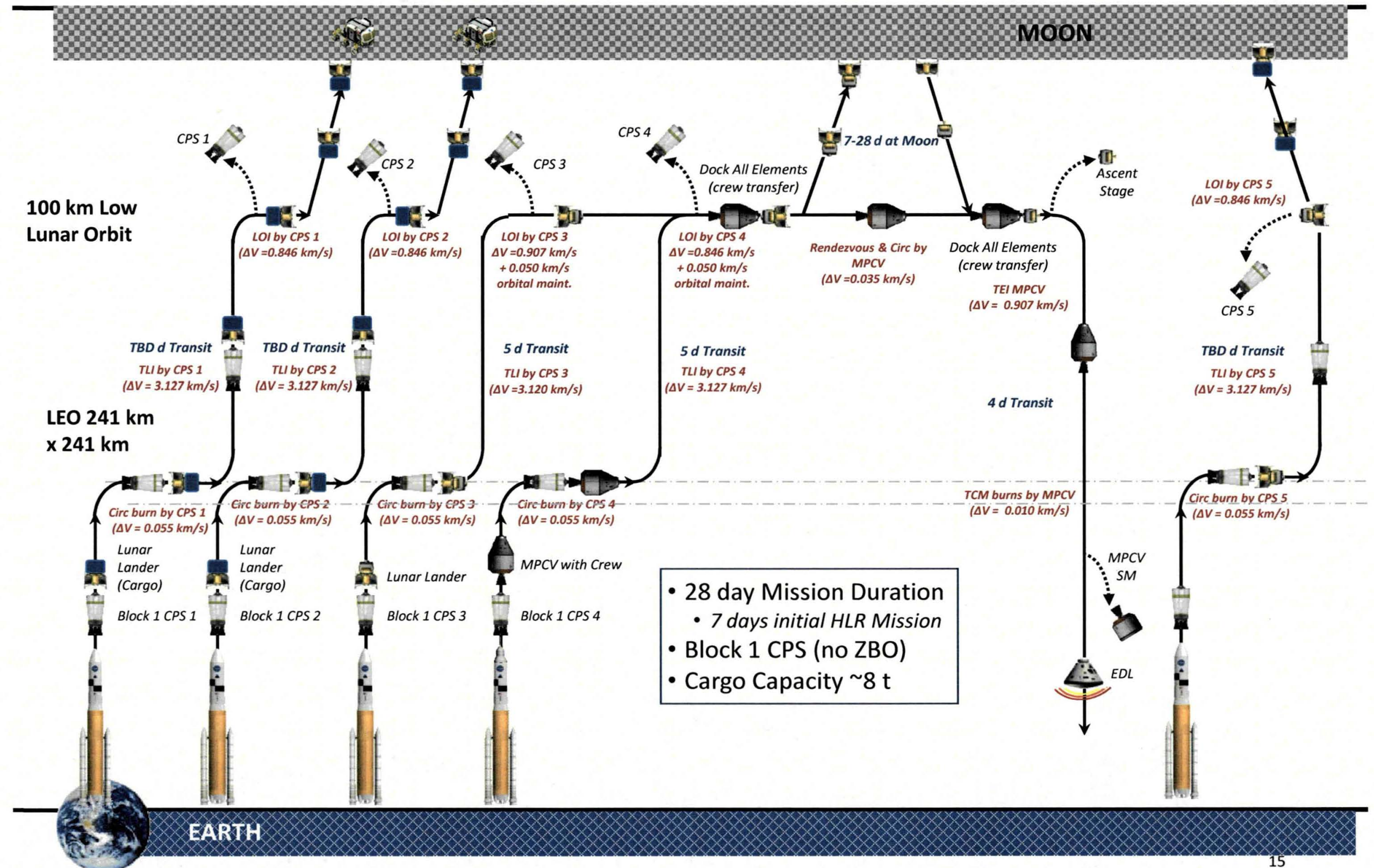




\section{Extended Stay and Surface Mobility Emphasis - GER}

4 large cargo landers, 6 small cargo landers, 5 crewed missions

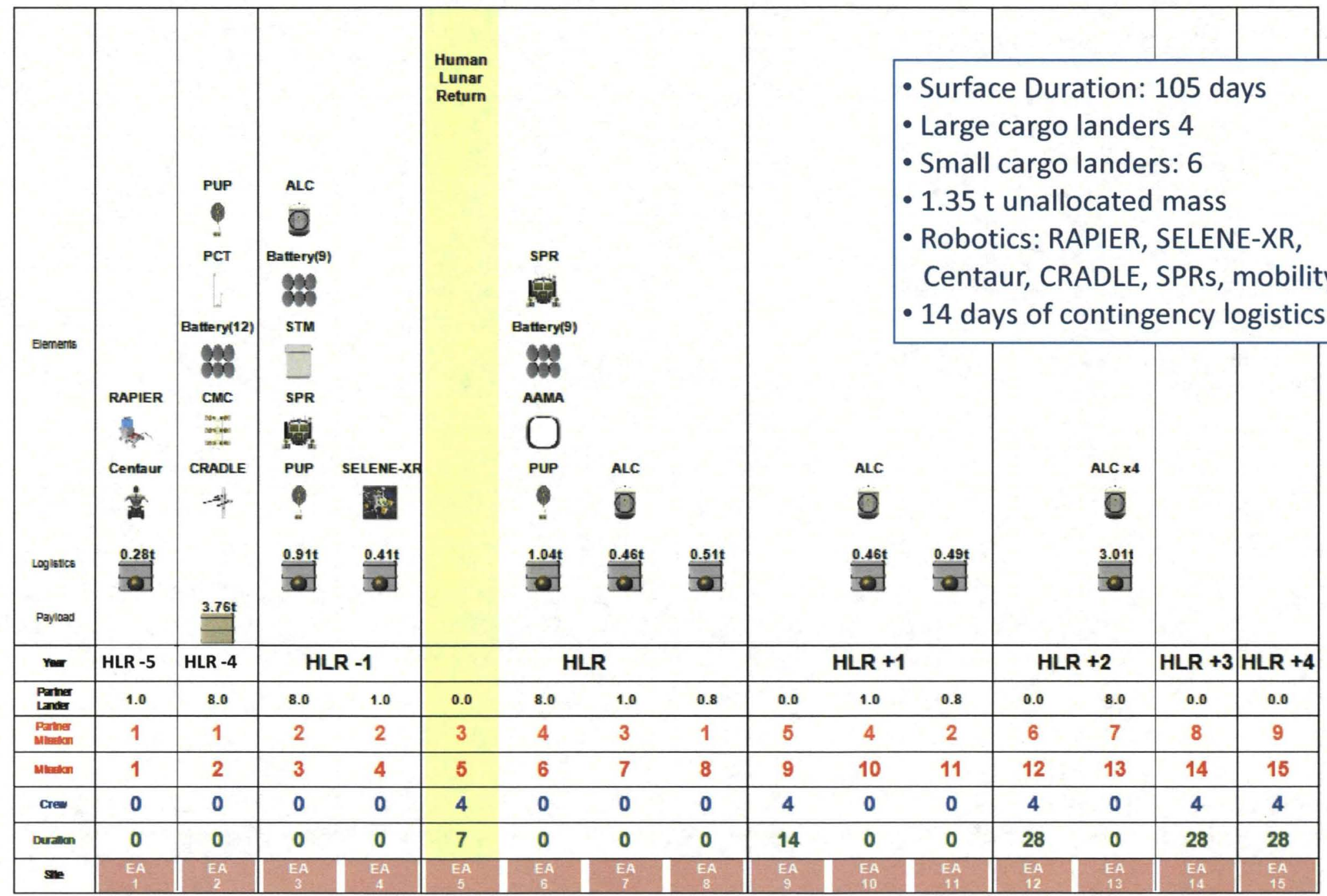




\section{GER Destination Elements}

\section{The GER DRM accumulates surface elements prior to the crew's arrival}

via a combination of small ( $\sim 1 \mathrm{mt})$ and large ( $8 \mathrm{mt})$ cargo landers

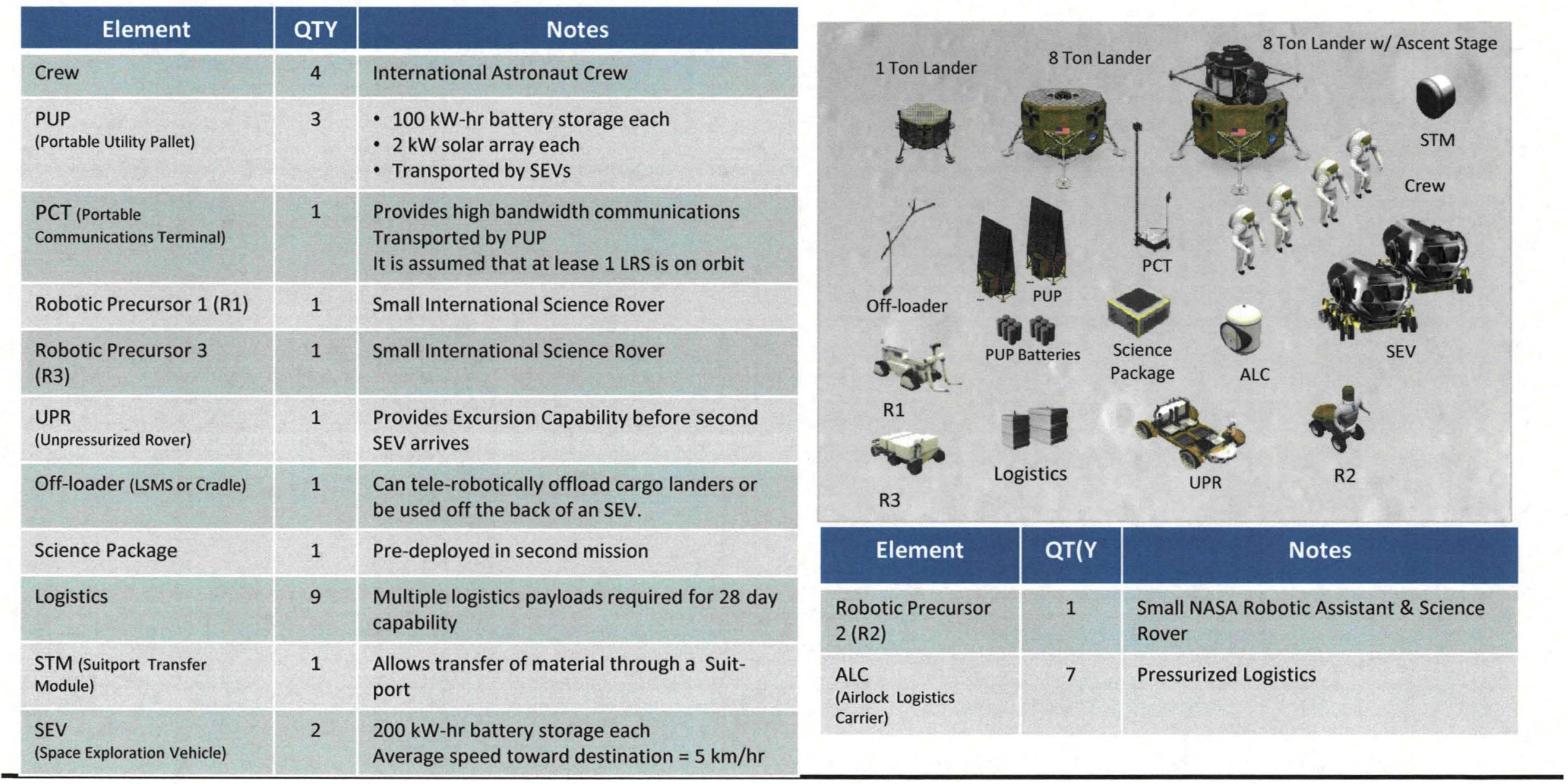

\section{Human Spaceflight Architecture Team}




\section{GER Extended Stay \& Surface Mobility Emphasis}

4 large cargo landers, 6 small cargo landers, 5 crewed missions

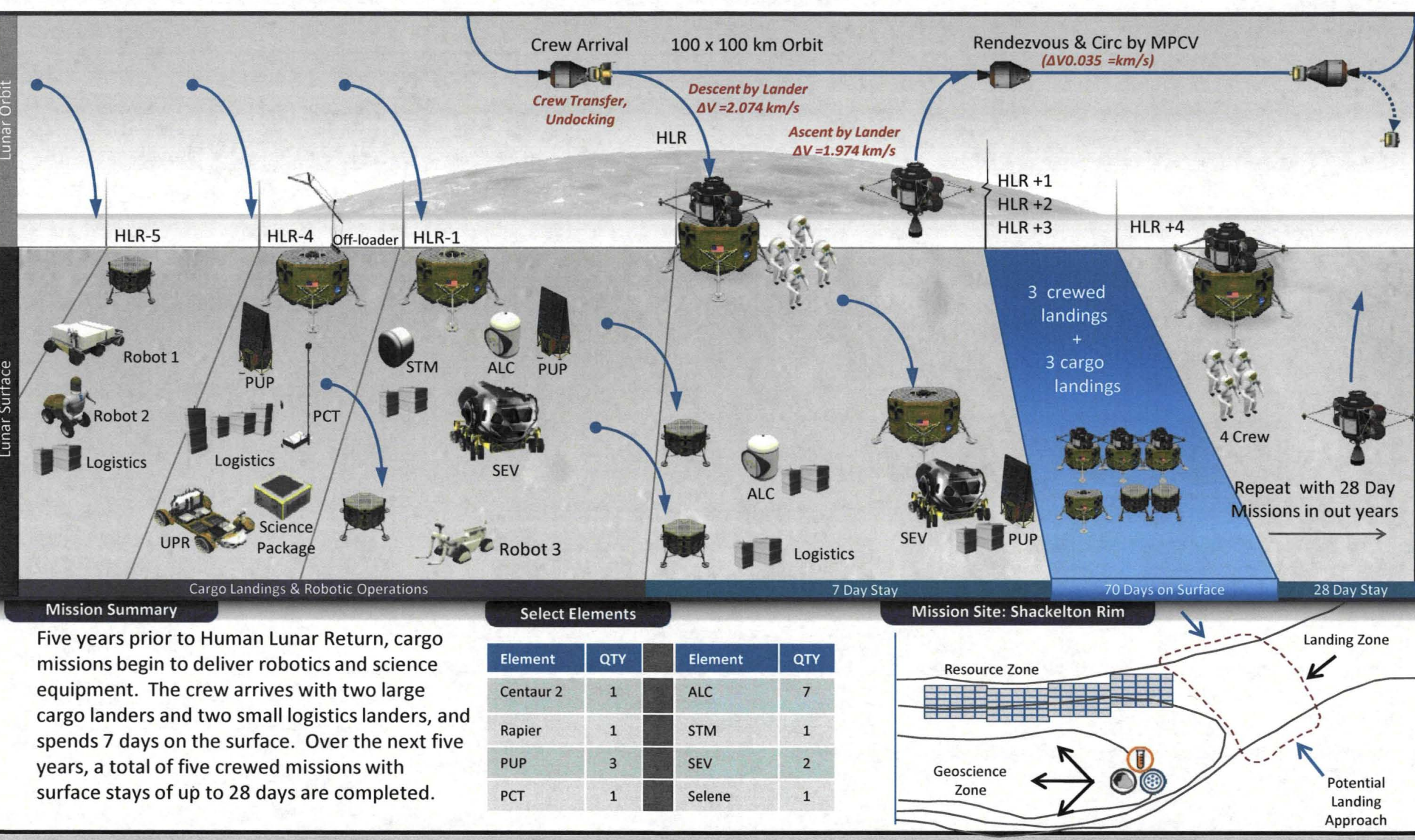

\section{Human Spaceflight Architecture Team}




\section{GER Lunar 7- 28 Day Extended Stay Con-Ops Overview}

\section{Extended Stay Polar Mission}

\section{$\underline{\text { Overview }}$}

An Extended Stay mission makes use of pre-deployed surface assets to extend the crewed surface stay beyond seven days. During the Cycle B excursion phases (polar only), these extended stay missions do not have large habitats to enable stays longer than 28 days. The crew will live in the SPRs which also provide mobility for excursions lasting up to 28 days depending on the available logistics supplies. The lander for these missions will not include an airlock. Crew egress and ingress from the ascent module is achieved through suitports. Space Exploration Vehicles (SEV) with Portable Utility Pallets (PUP) will provide all habitation and power during these missions.

- Human Lunar Return (HLR) Initial Operating Capability (IOC) = 7 days Mobile Excursions with 7 Days Contingency Logistics

- Crew lives in 2 SEV's and provide a mobile habitation capability at multiple landing sites

- IOC Mission achieves checkout of all systems and some Exploration and Science deployments

- Subsequent HLR +1 mission can achieve 14 days, while and HLR $+2,+3$, and +4 missions can achieve 28 days of operations with additional landed logistics

- The 28 day missions achieve long distance excursions and deploy science packages

- 28 Day Missions have 14 day contingencies

- The landing site for the 28 Day missions must remain in non-eclipse, sunlit areas while the SEVs and PUPS allow limited eclipse operations. 


\section{GER Lunar 7- 28 Day Extended Stay Con-Ops}

HLR-5: A small $1 \mathrm{t}$ class lander delivers several small robots to one of the lunar poles. These robots work together to identify a suitable landing site for an upcoming $8 \mathrm{t}$ human class lander. In addition to local reconnaissance, the small rovers gather science data, validate technologies and refine concurrent operations. This will be the first time ever that multiple robots will be working together in close proximity on another celestial body. The robots will practice servicing operations, scout the region for future crew/cargo landing areas, and deploy landing aides. All robots will send back to Earth a steady stream of engaging and informative data and video, including the descent and touchdown of future crewed/cargo landers. A crewed lunar flyby is performed, utilizing for the first time the cryogenic propulsion stage to leave the Earth's gravity well.

HLR-4: A year later, the human scale $8 \mathrm{t}$ lander touches down at the site identified by the small robots. It is carrying a version of the mobility chassis used by the crewed small pressurized rover. There is also the potential of it carrying small communications relay satellites that are deployed in lunar orbit to enable better coverage of the poles before descending. The mobility chassis will operate in autonomous and ground supervised modes at speeds and ranges far exceeding any previous planetary surface rover, and is outfitted with enough energy storage to survive the 14 day eclipse period. It will also be outfitted with hundreds of kilograms of science instruments and manipulators. The vast science payload, substantially increased speed and range along with the capability to survive lunar eclipse will allow it to traverse long distances away from the polar landing site to achieve regional exploration. In addition to its own science payloads, it will also be capable of transporting one or more of the previously delivered small robots as it explores which could be used for servicing and remote observation. This mobility platform will provide multiple partnering opportunities while substantially reducing risk for future exploration missions. 


\section{GER Lunar 7- 28 Day Extended Stay Con-Ops}

HLR-1: The lunar surface roving capability is augmented for a few years during this period by crewed missions to low lunar orbit that are testing the transportations system as well as refining techniques for tele-operating the surface assets from low lunar orbit. This activity is analogous to operating rovers on the surface of Mars from Mars orbit, as eventually may occur during a Mars orbital mission.

- The deployment of the pressurized rover and the crewed ascent module occur within a year of each other, thus increasing the potential to share systems development like ECLSS, thermal and power. Several years after the initial robotic missions, but before the first crewed mission, a small pressurized rover and supporting power infrastructure are landed in the polar region by a large cargo lander and self-deploy. It arrives on the surface as directed by the robotically emplaced landing aides.

- The small pressurized rover is initially tested, then sent on excursions (in a ground supervised mode) progressively further away from the landing location, beyond the range of the small robots, to identify opportunities and optimal paths that can be used by the humans on the first crewed mission.

- HLR: The humans arrive with any critical spares. The fully checked out rovers (original mobility chassis and the new small pressurized rover) are waiting for them. The crew then perform up to a 7 day mission, exploring the near polar region and practicing operations and contingency scenarios for upcoming traverses. Having two human scale rovers (one pressurized, one unpressurized) offers redundancy and rescue capabilities in the event one rover becomes non-operational. The crew leaves the surface at the end of their mission while the robots continue exploring before the next crew arrives, enhanced by portable utility pallets and cargo delivered by small international landers.

- Six months later another small pressurized rover is delivered, autonomously deployed and tested, so that it can join the previously delivered mobility chassis and pressurized rover at the next crewed landing location. 


\section{GER Lunar 7- 28 Day Extended Stay Con-Ops}

HLR+1: The next crew arrives one year after HLR performs a 14 day mission using the extended range and duration resulting from coupling the small pressurized rovers to the portable utility pallets. A crew does not return to this location for a year as the small pressurized rovers, the servicing robots and the portable utility pallets perform extensive ground supervised exploration

HLR+2, +3, +4: A third crewed mission arrives at the pole, with the goal of lengthening the mission duration to $\mathbf{2 8}$ days. This cycle repeats for two more years, with each mission lasting $\mathbf{2 8}$ days, enabled by the mobile infrastructure meeting the crew at new polar region landing sites and delivery of logistics and science instruments by small $1 \mathrm{t}$ landers.

By the time the mobile infrastructure is near the end of its design life, humans have spent 105 days on the lunar surface exploring and tested key planetary surface capabilities and operations. 